\title{
Synthesis, Antiprotozoal Activity, and Cheminformatic Analysis of 2-Phenyl-2H-Indazole Derivatives
}

\author{
Karen Rodríguez-Villar ${ }^{1}{ }^{(0)}$, Lilián Yépez-Mulia ${ }^{2}{ }^{(D}$, Miguel Cortés-Gines ${ }^{3}$, Jacobo David Aguilera-Perdomo ${ }^{3}$, \\ Edgar A. Quintana-Salazar ${ }^{3}$, Kevin Samael Olascoaga Del Angel ${ }^{4}$, Francisco Cortés-Benítez ${ }^{3}{ }^{1}$, \\ Juan Francisco Palacios-Espinosa ${ }^{3}$, Olivia Soria-Arteche ${ }^{3}$ and Jaime Pérez-Villanueva ${ }^{3, * \mathbb{D}}$ \\ 1 Doctorado en Ciencias Biológicas y de la Salud, Universidad Autónoma Metropolitana (UAM), \\ Ciudad de México 04960, Mexico; qkarenrodv@hotmail.com \\ 2 Unidad de Investigación Médica en Enfermedades Infecciosas y Parasitarias, UMAE Hospital de Pediatría, \\ Centro Médico Siglo XXI, Instituto Mexicano del Seguro Social, Ciudad de México 06720, Mexico; \\ lilianyepez@yahoo.com \\ 3 Departamento de Sistemas Biológicos, División de Ciencias Biológicas y de la Salud, \\ Universidad Autónoma Metropolitana-Xochimilco (UAM-X), Ciudad de México 04960, Mexico; \\ mcortes@pharmometrica.com.mx (M.C.-G.); jacoboaguilera.96@gmail.com (J.D.A.-P.); \\ edgarqs12811@gmail.com (E.A.Q.-S.); jcortesb@correo.xoc.uam.mx (F.C.-B.); \\ jpalacios@correo.xoc.uam.mx (J.F.P.-E.); soriao@correo.xoc.uam.mx (O.S.-A.) \\ 4 Doctorado en Biología Experimental, Universidad Autónoma Metropolitana (UAM), \\ Ciudad de México 04960, Mexico; olaskuaga@gmail.com \\ check for \\ updates \\ * Correspondence: jpvillanueva@correo.xoc.uam.mx; Tel.: +525-54-83-72-59; Fax: +525-55-94-79-29
}

Citation: Rodríguez-Villar, K.; Yépez-Mulia, L.; Cortés-Gines, M.; Aguilera-Perdomo, J.D.; Quintana-Salazar, E.A.; Olascoaga Del Angel, K.S.; Cortés-Benítez, F.; Palacios-Espinosa, J.F.; Soria-Arteche, O.; Pérez-Villanueva, J. Synthesis, Antiprotozoal Activity, and Cheminformatic Analysis of 2-Phenyl-2H-Indazole Derivatives. Molecules 2021, 26, 2145. https:// doi.org/10.3390/molecules26082145

Academic Editor: Josef Jampilek

Received: 28 February 2021

Accepted: 6 April 2021

Published: 8 April 2021

Publisher's Note: MDPI stays neutral with regard to jurisdictional claims in published maps and institutional affiliations.

Copyright: (c) 2021 by the authors. Licensee MDPI, Basel, Switzerland. This article is an open access article distributed under the terms and conditions of the Creative Commons Attribution (CC BY) license (https:// creativecommons.org/licenses/by/ $4.0 /)$.
Abstract: Indazole is an important scaffold in medicinal chemistry. At present, the progress on synthetic methodologies has allowed the preparation of several new indazole derivatives with interesting pharmacological properties. Particularly, the antiprotozoal activity of indazole derivatives have been recently reported. Herein, a series of 22 indazole derivatives was synthesized and studied as antiprotozoals. The 2-phenyl-2H-indazole scaffold was accessed by a one-pot procedure, which includes a combination of ultrasound synthesis under neat conditions as well as Cadogan's cyclization. Moreover, some compounds were derivatized to have an appropriate set to provide structure-activity relationships (SAR) information. Whereas the antiprotozoal activity of six of these compounds against E. histolytica, G. intestinalis, and T. vaginalis had been previously reported, the activity of the additional 16 compounds was evaluated against these same protozoa. The biological assays revealed structural features that favor the antiprotozoal activity against the three protozoans tested, e.g., electron withdrawing groups at the 2-phenyl ring. It is important to mention that the indazole derivatives possess strong antiprotozoal activity and are also characterized by a continuous SAR.

Keywords: Entamoeba histolytica; Giardia intestinalis; Trichomonas vaginalis; indazole; structureactivity relationships

\section{Introduction}

Indazole is an important scaffold in medicinal chemistry. So far, a few drugs containing the indazole moiety are currently available for clinical use; for instance, the antiemetic granisetron, the nonsteroidal anti-inflammatory drugs benzydamine and bendazac, the anticancer agents pazopanib, axitinib, niraparib and entrectinib. From these, niraparib and entrectinib were recently approved in 2017 and 2019 respectively [1,2]. At present, the progress on synthetic methodologies has allowed the preparation of several new indazole derivatives with interesting pharmacological properties [3-6]. Particularly, the antiprotozoal activity of indazole derivatives against Entamoeba histolytica [7], Giardia intestinalis and Trichomonas vaginalis have been recently reported $[8,9]$. 
E. histolytica and G. intestinalis are intestinal protozoa, which causes amebiasis and giardiasis, respectively. Worldwide 50 million amebiasis cases and 100,000 deaths annually are estimated [10], whereas 280 million people are affected by G. intestinalis [11]. Both diseases have high impact in the public health, causing diarrhea, which is a major factor in morbidity and mortality, affecting mainly infant population [12]. On the other hand, trichomoniasis, caused by $T$. vaginalis is the most common non-viral sexually transmitted disease [13]. According to the World Health Organization, more than 156 million new cases are estimated annually worldwide [14]. Trichomoniasis can be the cause of cervicitis, urethritis, vaginitis, and genital ulceration; additionally, it has been associated with preterm labor, low-birth weight, sterility, cervical cancer, and a predisposition to HIV infection [14-16]. Although some chemotherapeutic agents are available to treat amebiasis, giardiasis and trichomoniasis, e.g., nitroderivatives such as metronidazole $[13,16,17]$, the increased drug resistance [13,16-19], and absence of new approved drugs with alternative mechanism of action, lead us to search new antiprotozoal compounds, particularly based on the indazole scaffold. Herein, 22 indazole derivatives were synthesized employing a practical one-pot process. Whereas six of these compounds have been previously reported to have activity against E. histolytica, G. intestinalis, and T. vaginalis, in this study, the activity of the additional 16 compounds against these three parasites was evaluated. Moreover, a SAR analysis and activity landscape studies were performed to highlight relevant structural features for the biological activity and to identify the SAR nature of the compounds studied.

\section{Results and Discussion}

\subsection{Synthesis of 2-Phenyl-2H-Indazole Derivatives}

The synthetic route for the synthesis of 2-phenyl-2H-indazole derivatives is shown in Scheme 1. For this purpose, a new practical modification of the Cadogan's method using an ultrasound assisted one-pot synthesis was employed. This method uses a combination of ultrasound synthesis as previously described by Crawford under neat conditions as well as the Cadogan's cyclization by refluxing the Schiff base with triethyl phosphite [20,21]. A comparison of the employed one-pot procedure and a previously reported methodology for representative examples is displayed in Table 1. Noteworthy, the one-pot method leads to similar or better yields than our previous report [8]. Moreover, it is important to mention that our method avoids one purification step, save solvents, and reduce the work up time. To expand the scope of the method, the number of compounds available for biological assays, and the diversity of substituents for SAR analysis, derivatives with chlorine, methoxy, methoxycarbonyl, fluorine and trifluoromethyl groups at the 2- or 3positions of the phenyl substituent were also synthesized. It is to be noted that compound 18, having a 2-(methoxycarbonyl)phenyl substituent, was not obtained by employing the described method, instead, 18a having a 2-cyanophenyl was synthesized as alternative intermediary to obtain 18; however a slight method modification was also needed to achieve the compound 18a (vide infra). Since compounds substituted with $\mathrm{OH}$ and $\mathrm{COOH}$ are important from the medicinal chemistry point of view due to the fact that it forms key protein-ligand interactions (e.g., hydrogen bonds and salt bridges) [22], derivatives having hydroxyl $(7,14$ and 21$)$ and carboxyl $(8,15$ and 22) groups were synthesized by hydrolysis of precursors 3,10, 17, 4, 11 and 18a. All synthesized compounds were characterized by ${ }^{1} \mathrm{H}$ NMR and ${ }^{13} \mathrm{C}$ NMR spectra and the new structures were also characterized by mass spectrometry. The nuclear magnetic resonance spectra of all compounds can be found in Figures S1-S23 in the Supplementary Materials. 
<smiles>O=Cc1ccccc1[N+](=O)[O-]</smiles><smiles>[R]c1ccc(-n2cc3ccccc3n2)c([R])c1[R]</smiles>

1: $\mathrm{R}^{1}=\mathrm{H}, \mathrm{R}^{2}=\mathrm{H}, \mathrm{R}^{3}=\mathrm{H}$

2: $\mathrm{R}^{1}=\mathrm{Cl}, \mathrm{R}^{2}=\mathrm{H}, \mathrm{R}^{3}=\mathrm{H}$

3: $\mathrm{R}^{1}=\mathrm{OCH}_{3}, \mathrm{R}^{2}=\mathrm{H}, \mathrm{R}^{3}=\mathrm{H}$

4: $\mathrm{R}^{1}=\mathrm{COOCH}_{3}, \mathrm{R}^{2}=\mathrm{H}, \mathrm{R}^{3}=\mathrm{H}$

5: $R^{1}=F, R^{2}=H, R^{3}=H$

6: $\mathrm{R}^{1}=\mathrm{CF}_{3}, \mathrm{R}^{2}=\mathrm{H}, \mathrm{R}^{3}=\mathrm{H}$

9: $\mathrm{R}^{1}=\mathrm{H}, \mathrm{R}^{2}=\mathrm{Cl}, \mathrm{R}^{3}=\mathrm{H}$

10: $R^{1}=H, R^{2}=O_{3}, R^{3}=H$

11: $R^{1}=H, R^{2}=\mathrm{COOCH}_{3}, R^{3}=H$

12: $R^{1}=H, R^{2}=F, R^{3}=H$

13: $R^{1}=H, R^{2}=C F_{3}, R^{3}=H$

16: $R^{1}=H, R^{2}=H, R^{3}=C l$

17: $R^{1}=H, R^{2}=H, R^{3}=\mathrm{OCH}_{3}$

18a: $R^{1}=H, R^{2}=H, R^{3}=C N$

19: $R^{1}=H, R^{2}=H, R^{3}=F$

20: $R^{1}=H, R^{2}=H, R^{3}=C F_{3}$<smiles>[R]c1ccc(-n2cc3ccccc3n2)c([R])c1[R]</smiles>

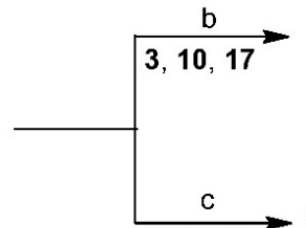

7: $\mathrm{R}^{1}=\mathrm{OH}, \mathrm{R}^{2}=\mathrm{H}, \mathrm{R}^{3}=\mathrm{H}$

14: $\mathrm{R}^{1}=\mathrm{H}, \mathrm{R}^{2}=\mathrm{OH}, \mathrm{R}^{3}=\mathrm{H}$

21: $R^{1}=H, R^{2}=H, R^{3}=O H$

$4,11,18 a$

8: $\mathrm{R}^{1}=\mathrm{COOH}, \mathrm{R}^{2}=\mathrm{H}, \mathrm{R}^{3}=\mathrm{H}$

15: $R^{1}=H, R^{2}=\mathrm{COOH}, \mathrm{R}^{3}=\mathrm{H}$

d

22: $R^{1}=H, R^{2}=H, R^{3}=C O O H$

18: $R^{1}=H, R^{2}=H, R^{3}=\mathrm{COOCH}_{3}$

Scheme 1. Synthesis of the 2-phenylindazole derivatives. Reagents and conditions: (a) $1 . \mathrm{R}^{1} \mathrm{C}_{6} \mathrm{H}_{4} \mathrm{NH}_{2}, \mathrm{ultrasound}, 40{ }^{\circ} \mathrm{C}$, neat, 2. $\mathrm{P}(\mathrm{OEt})_{3}$, reflux; (b) $\mathrm{BBr}_{3}, \mathrm{CH}_{2} \mathrm{Cl}_{2}, 0^{\circ} \mathrm{C}$; (c) 1 . $\mathrm{NaOH}, \mathrm{H}_{2} \mathrm{O}$, reflux, 2. $\mathrm{HCl}$; (d) $\mathrm{HCl}, \mathrm{MeOH}, \mathrm{MW}$.

Table 1. Chemical yield (\%) for 2-phenyl-2H-indazole derivatives synthesized by ultrasound assisted one-pot procedure, and representative examples of these same compounds synthesized by the conventional method.

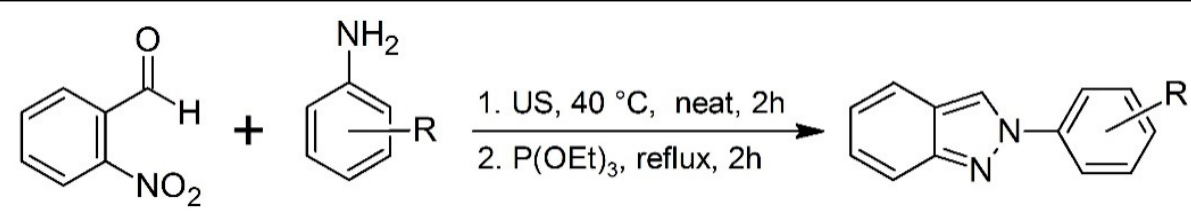

\begin{tabular}{cccc}
\hline Compound & $\mathbf{R}$ & $\begin{array}{c}\text { Ultrasound Assisted } \\
\text { One-Pot Procedure }\end{array}$ & $\begin{array}{c}\text { Previously Reported by the } \\
\text { Conventional Method [8] }\end{array}$ \\
\hline $\mathbf{1}$ & $\mathrm{H}$ & 92 & 60 \\
$\mathbf{2}$ & $4-\mathrm{Cl}$ & 62 & 52 \\
$\mathbf{3}$ & $4-\mathrm{OCH}_{3}$ & 57 & 52 \\
$\mathbf{4}$ & $4-\mathrm{COOCH}_{3}$ & 39 & 38 \\
$\mathbf{5}$ & $4-\mathrm{F}$ & 71 & 50 \\
$\mathbf{6}$ & $4-\mathrm{CF}_{3}$ & 43 & - \\
$\mathbf{9}$ & $3-\mathrm{Cl}_{3}$ & 53 & - \\
$\mathbf{1 0}$ & $3-\mathrm{OCH}_{3}$ & 45 & - \\
$\mathbf{1 1}$ & $3-\mathrm{COOCH}_{3}$ & 51 & - \\
$\mathbf{1 2}$ & $3-\mathrm{F}$ & 51 & - \\
$\mathbf{1 3}$ & $3-\mathrm{CF}_{3}$ & 46 & - \\
$\mathbf{1 6}$ & $2-\mathrm{Cl}$ & 55 & - \\
$\mathbf{1 7}$ & $2-\mathrm{OCH}_{3}$ & 69 & - \\
$\mathbf{1 8}$ & $2-\mathrm{CN}_{\mathbf{1 9}}$ & 321 & - \\
$\mathbf{2 0}$ & $2-\mathrm{F}$ & 54 & - \\
\hline
\end{tabular}

${ }^{1}$ The reaction mixture was ultrasonicated by four cycles of $1 \mathrm{~h}$ at $40^{\circ} \mathrm{C}$ (vide infra). 


\subsection{Antiprotozoal Activity}

Compounds 5, 6, 9-22 were tested in vitro against $E$. histolytica, $G$. intestinalis, and $T$. vaginalis. It is important to mention that the activity of compounds 1-4, $\mathbf{7}$ and $\mathbf{8}$ against these three protozoa were previously reported by our group [8], however, the values were included in Table 2 in order to support the SAR analysis. Against E. histolytica, the first interesting finding was that all tested compounds were more potent than $1 \mathrm{H}$-indazole reported by López-Vallejo et al. $\left(\mathrm{IC}_{50}=0.740 \mu \mathrm{M}\right.$ ) [7]. It is important to note that biological assays were performed using the same strain, under the same conditions as well as the same laboratory. The results suggest that 2-phenyl substitution is important for the antiprotozoal activity, since this modification increased 9-fold the activity. The best potency was found for 2-phenyl-2H-indazole derivatives substituted with methoxycarbonyl $\left(4,11\right.$ and 18), 4-chlorophenyl (2) and 2-(trifluoromethyl)phenyl (20) $\left(\mathrm{IC}_{50}<0.050\right.$ $\mu \mathrm{M})$. On the other hand, the results for the assays performed against $G$. intestinalis showed that the derivatives substituted with 2-chlorophenyl (16), 2-(methoxycarbonyl)phenyl (18), 2-(trifluoromethyl)phenyl (20) and 2-carboxyphenyl (22) displayed the best activity $\left(\mathrm{IC}_{50}<0.050 \mu \mathrm{M}\right)$. Moreover, 4- and 3-(trifluoromethyl)phenyl derivatives (6 and 13 respectively) showed favorable effect on the giardicidal activity. It is worth to emphasize that compounds were slightly more potent against E. histolytica and G. intestinalis than T. vaginalis. Hence, against $T$. vaginalis, the best activity $\left(\mathrm{IC}_{50}<0.070 \mu \mathrm{M}\right)$ was found for the derivatives substituted with 3-(methoxycarbonyl)phenyl (11), 3-(trifluoromethyl)phenyl (13) and 3-carboxyphenyl (15), as well as the 2-chlorophenyl (16) and 2-carboxyphenyl (22). The $\mathrm{IC}_{50}$ values and SAR data displayed in Figure 1, indicate that electron-withdrawing substituents attached to the 2-phenyl ring are favorable for the antiprotozoal activity against the three evaluated protozoa.

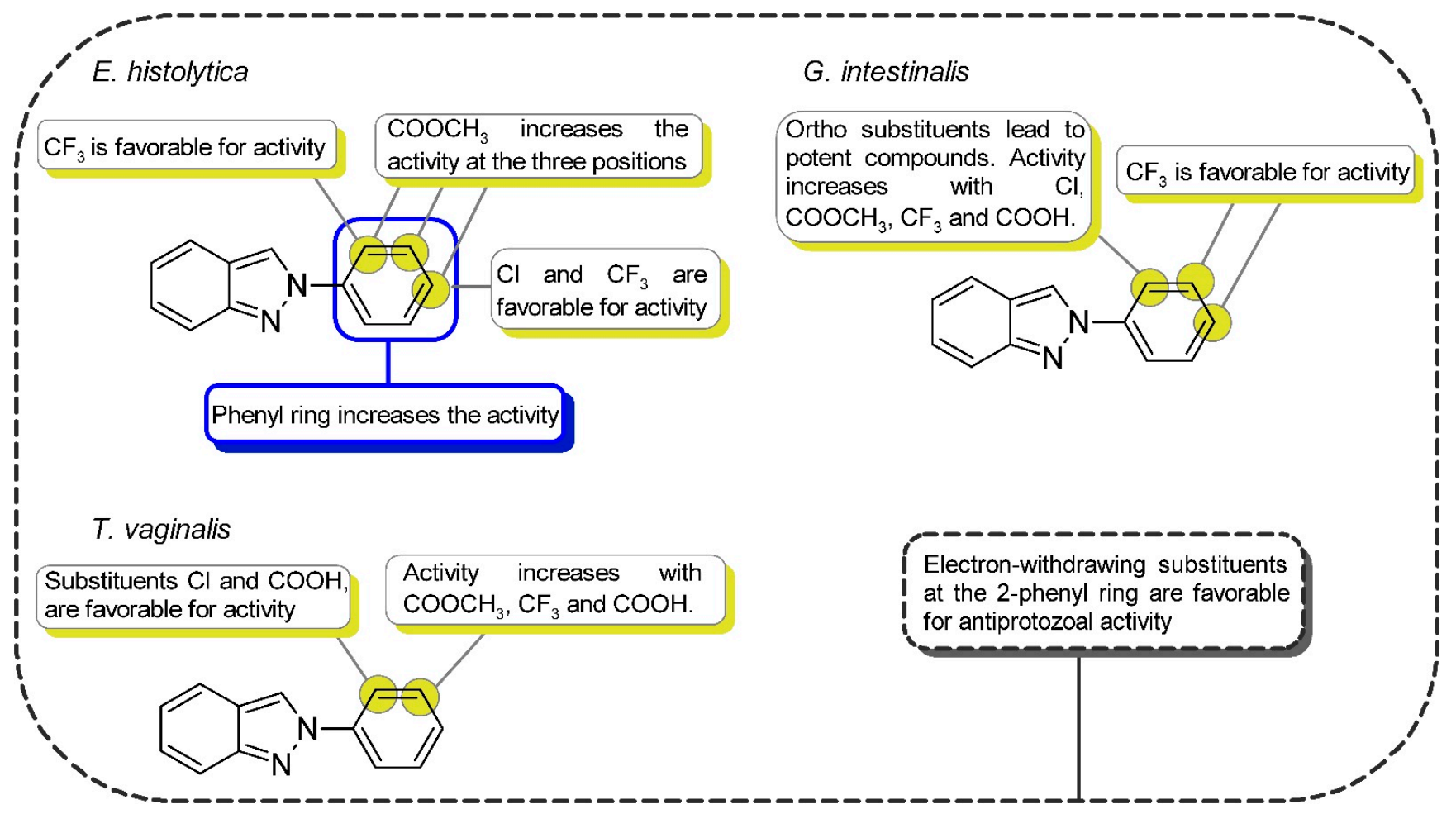

Figure 1. Principally SAR features derived from the 2-phenyl- $2 \mathrm{H}$-indazole derivatives tested. 
Table 2. Antiprotozoal activity of the 2-pheny-2H-indazole derivatives synthesized $\left(\mathrm{IC}_{50}[\mu \mathrm{M}] \pm \mathrm{SD}\right)$.

\begin{tabular}{|c|c|c|c|c|c|c|}
\hline Compound & $\mathbf{R}^{1}$ & $\mathbf{R}^{2}$ & $\mathbf{R}^{3}$ & E. histolytica & G. intestinalis & T. vaginalis \\
\hline $\mathbf{1}^{1}$ & $\mathrm{H}$ & $\mathrm{H}$ & $\mathrm{H}$ & $0.080 \pm 0.004$ & $0.113 \pm 0.022$ & $0.118 \pm 0.022$ \\
\hline $2^{1}$ & $\mathrm{Cl}$ & $\mathrm{H}$ & $\mathrm{H}$ & $0.042 \pm 0.003$ & $0.063 \pm 0.003$ & $0.107 \pm 0.003$ \\
\hline $3^{1}$ & $\mathrm{OCH}_{3}$ & $\mathrm{H}$ & $\mathrm{H}$ & $0.154 \pm 0.016$ & $0.205 \pm 0.006$ & $0.372 \pm 0.016$ \\
\hline $4^{1}$ & $\mathrm{COOCH}_{3}$ & $\mathrm{H}$ & $\mathrm{H}$ & $0.022 \pm 0.003$ & $0.063 \pm 0.006$ & $0.107 \pm 0.006$ \\
\hline 5 & F & $\mathrm{H}$ & $\mathrm{H}$ & $0.075 \pm 0.007$ & $0.071 \pm 0.007$ & $0.118 \pm 0.020$ \\
\hline 6 & $\mathrm{CF}_{3}$ & $\mathrm{H}$ & $\mathrm{H}$ & $0.053 \pm 0.000$ & $0.034 \pm 0.005$ & $0.084 \pm 0.016$ \\
\hline $7^{1}$ & $\mathrm{OH}$ & $\mathrm{H}$ & $\mathrm{H}$ & $0.074 \pm 0.010$ & $0.119 \pm 0.007$ & $0.157 \pm 0.014$ \\
\hline $8^{1}$ & $\mathrm{COOH}$ & $\mathrm{H}$ & $\mathrm{H}$ & $0.097 \pm 0.006$ & $0.193 \pm 0.012$ & $0.327 \pm 0.018$ \\
\hline 9 & $\mathrm{H}$ & $\mathrm{Cl}$ & $\mathrm{H}$ & $0.070 \pm 0.012$ & $0.083 \pm 0.012$ & $0.101 \pm 0.006$ \\
\hline 10 & $\mathrm{H}$ & $\mathrm{OCH}_{3}$ & $\mathrm{H}$ & $0.078 \pm 0.010$ & $0.056 \pm 0.010$ & $0.120 \pm 0.019$ \\
\hline 11 & $\mathrm{H}$ & $\mathrm{COOCH}_{3}$ & $\mathrm{H}$ & $0.036 \pm 0.000$ & $0.054 \pm 0.003$ & $0.069 \pm 0.008$ \\
\hline 12 & $\mathrm{H}$ & F & $\mathrm{H}$ & $0.097 \pm 0.010$ & $0.087 \pm 0.003$ & $0.092 \pm 0.010$ \\
\hline 13 & $\mathrm{H}$ & $\mathrm{CF}_{3}$ & $\mathrm{H}$ & $0.061 \pm 0.005$ & $0.050 \pm 0.005$ & $0.069 \pm 0.005$ \\
\hline 14 & $\mathrm{H}$ & $\mathrm{OH}$ & $\mathrm{H}$ & $0.102 \pm 0.003$ & $0.105 \pm 0.000$ & $0.162 \pm 0.000$ \\
\hline 15 & $\mathrm{H}$ & $\mathrm{COOH}$ & $\mathrm{H}$ & $0.074 \pm 0.009$ & $0.053 \pm 0.009$ & $0.065 \pm 0.009$ \\
\hline 16 & $\mathrm{H}$ & $\mathrm{H}$ & $\mathrm{Cl}$ & $0.088 \pm 0.006$ & $0.031 \pm 0.006$ & $0.066 \pm 0.012$ \\
\hline 17 & $\mathrm{H}$ & $\mathrm{H}$ & $\mathrm{OCH}_{3}$ & $0.096 \pm 0.003$ & $0.083 \pm 0.003$ & $0.183 \pm 0.006$ \\
\hline 18 & $\mathrm{H}$ & $\mathrm{H}$ & $\mathrm{COOCH}_{3}$ & $0.050 \pm 0.003$ & $0.032 \pm 0.006$ & $0.109 \pm 0.008$ \\
\hline 19 & $\mathrm{H}$ & $\mathrm{H}$ & $\mathrm{F}$ & $0.092 \pm 0.010$ & $0.071 \pm 0.007$ & $0.108 \pm 0.007$ \\
\hline 20 & $\mathrm{H}$ & $\mathrm{H}$ & $\mathrm{CF}_{3}$ & $0.031 \pm 0.011$ & $0.027 \pm 0.011$ & $0.128 \pm 0.014$ \\
\hline 21 & $\mathrm{H}$ & $\mathrm{H}$ & $\mathrm{OH}$ & $0.145 \pm 0.010$ & $0.074 \pm 0.003$ & $0.112 \pm 0.010$ \\
\hline 22 & $\mathrm{H}$ & $\mathrm{H}$ & $\mathrm{COOH}$ & $0.074 \pm 0.003$ & $0.023 \pm 0.009$ & $0.067 \pm 0.006$ \\
\hline $\mathrm{MTZ}^{2}$ & - & - & - & $0.380 \pm 0.146$ & $1.226 \pm 0.125$ & $0.236 \pm 0.016$ \\
\hline $\mathrm{ABZ}^{3}$ & - & - & - & $56.533 \pm 18.845$ & $0.037 \pm 0.003$ & $1.591 \pm 0.011$ \\
\hline
\end{tabular}

${ }^{1} \mathrm{IC}_{50}$ values were taken from Pérez-Villanueva et al. 2017 [8]; ${ }^{2}$ Metronidazole; ${ }^{3}$ Albendazole.

\subsection{Cheminformatic Analysis}

Compounds 1-22 were also analyzed employing cheminformatic tools and compared against antiprotozoal databases of benzimidazole derivatives $[7,23-36]$ and ChEMBL compounds [37], which have also reported activities against E. histolytica, G. intestinalis and T. vaginalis. It is worthwhile to mention that benzimidazole derivatives compose an important reference database since a high number of compounds were tested under the same method and conditions. These benzimidazole derivatives have been the reference database for several cheminformatic and QSAR studies as antiprotozoals [31,34,38-40]; and the data available has been expanded during the last years. Figure 2 shows a representation of the property space and the activity distribution of the 22 synthesized indazole derivatives as compared to benzimidazole derivatives and ChEMBL collection for each analyzed parasite [41]. Although, 2-phenyl-2H-indazole derivatives are localized in a very focalized space as compared to benzimidazole and ChEMBL compounds, their activity values are similar to the majority of benzimidazoles, but higher than ChEMBL compounds in most cases, as is visually depicted as violin plots in Figure 2. Although the number and diversity of indazole derivatives synthesized and tested herein are still low, it is of our interest to expand the database of indazole derivatives as well as their activity landscape to search for more potent compounds. 
E. histolytica
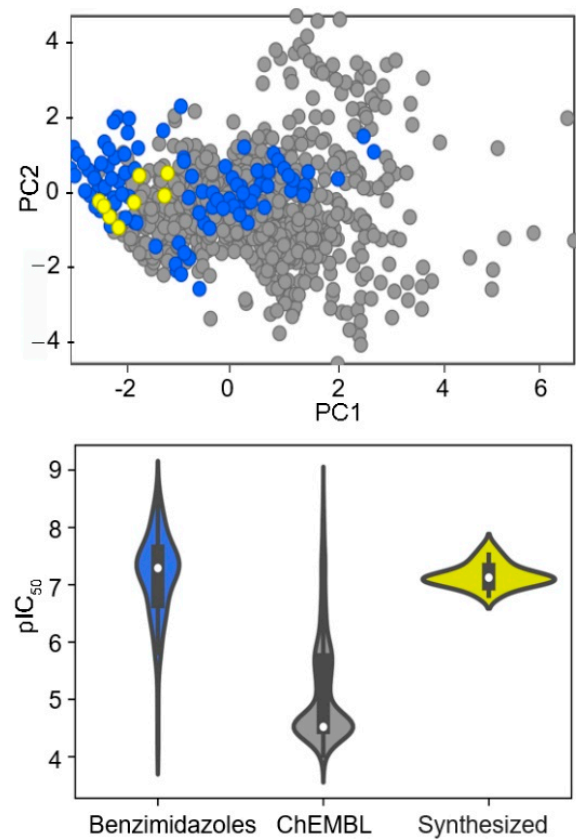

G. intestinalis
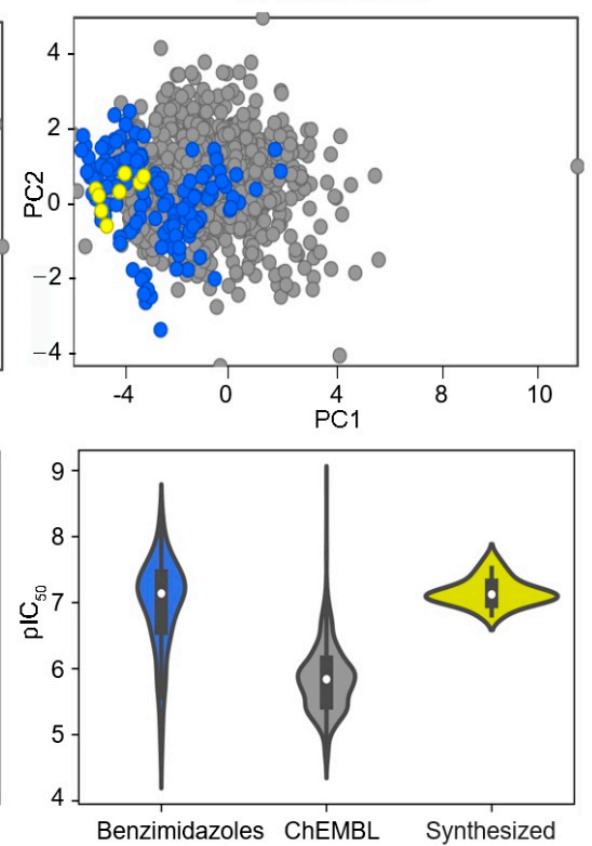

T. vaginalis
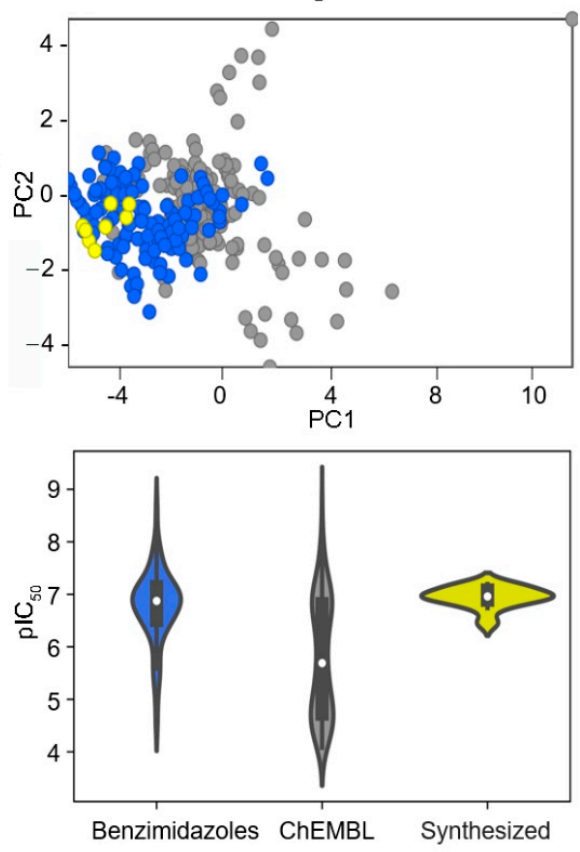

Figure 2. Property space and activity range for 2-phenyl-2H-indazole derivatives (1-22) synthesized in this work (yellow), a collection of antiprotozoal $1 \mathrm{H}$-benzimidazole derivatives (blue) and ChEMBL collection of antiprotozoals (gray). The two principal components taken together account for $75.34,72.44$ and $75.71 \%$ of the variance for E. histolytica, G. intestinalis, and T. vaginalis, respectively.

To visualize the activity landscape of the synthesized indazole derivatives as compared to the benzimidazole reference database, chemotype-based Structure-Activity Similarity (SAS) maps were generated (Figure 3) [42]. A general form of the SAS map is presented in Figure 4. SAS maps are divided in four regions: region I is associated with scaffold hopping (or side chain hopping), region II denotes smooth SAR and region IV indicates discontinuous SAR and activity cliffs. The structure similarity threshold was established by the median similarity of the pairwise comparisons plotted in each map and depends on the database and the fingerprint employed, whereas the activity similarity threshold was established on two units in activity difference (100-fold difference in potency). Since Similarity-Property principle establish that similar compounds have similar properties (e.g., biological activity), all indazole derivatives fall in such definition [43]. Different SAR classifications have been previously defined as continuous (smooth), heterogeneous and discontinuous (rough) SAR [43,44]. Moreover, the implications in medicinal chemistry, disadvantages or opportunities implied on different SAR classification has been widely discussed $[44,45]$. Therefore, the 22 indazole derivatives studied are characterized by a smooth or continuous SAR (high similarity and low activity differences; region II) as compared to the benzimidazole derivatives database where a heterogeneous SAR can be observed [38,39]. Therefore, the antiprotozoal indazole database should be expanded by including more diverse substituted derivatives at different positions to carry out QSAR studies and lead optimization. 

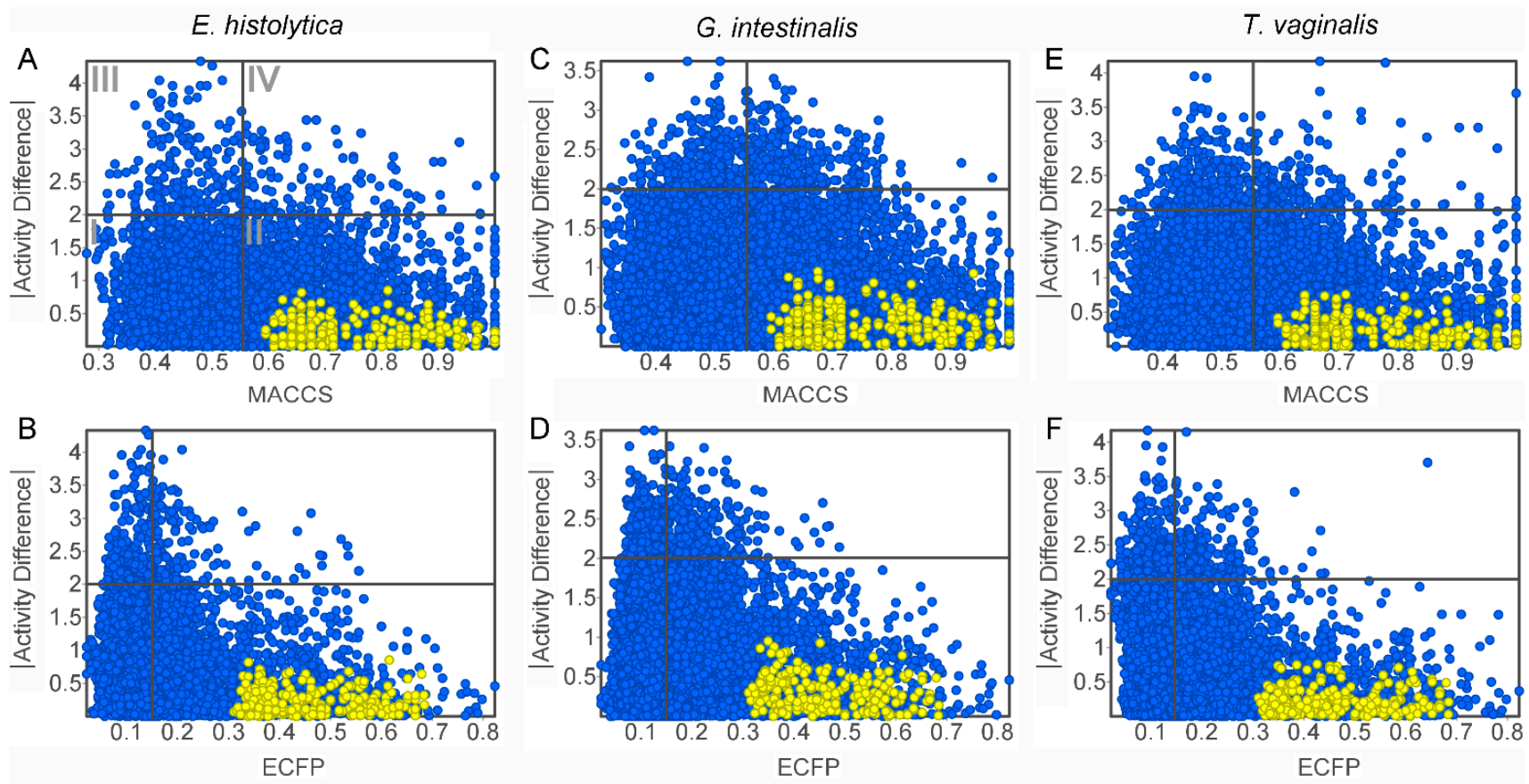

Figure 3. Structure-Activity Similarity Maps using Molecular ACCess System keys (MACCS) and Extended Connectivity Fingerprint (ECFP) divided by thresholds in structure similarity and activity differences in regions I-IV. Each data point indicates a pairwise comparison of the 22 synthesized 2-phenyl-2H-indazole derivatives (231 datapoints in yellow, A-F) and 111 benzimidazoles tested against E. histolytica (6105 datapoints in blue, A,B), 159 benzimidazoles tested against $G$. intestinalis (12,561 datapoints in blue, C,D), and 134 benzimidazoles tested against T. vaginalis (8911 datapoints in blue, E,F).

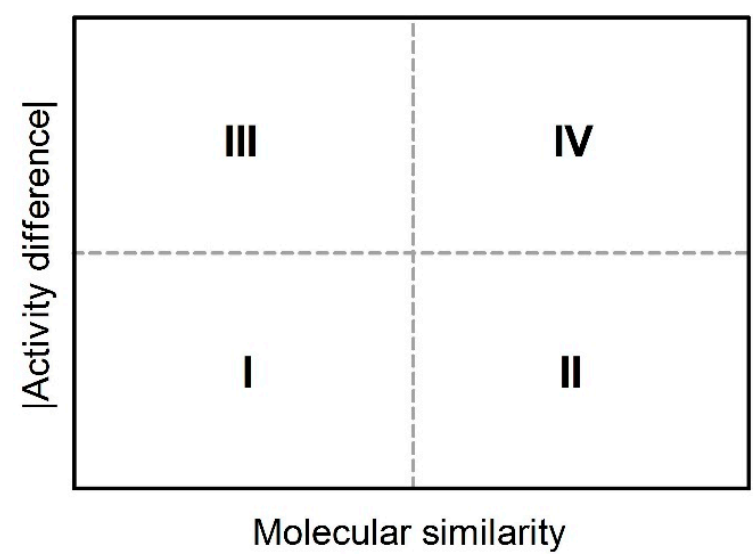

Figure 4. General form of the structure-activity similarity (SAS) maps showing four major regions.

\section{Materials and Methods}

\subsection{Chemicals and Instruments}

All chemicals and starting materials were obtained from Sigma-Aldrich (SigmaAldrich, Toluca, MEX, Mexico). Reactions were monitored by TLC on $0.2 \mathrm{~mm}$ percolated silica gel $60 \mathrm{~F}_{254}$ plates (Merck, Darmstadt, Germany) and visualized by irradiation with a UV lamp (Cole-Parmer, Vernon Hills, IL, USA). Silica gel 60, 70-230 mesh, was used for column chromatography (Macherey-Nagel, Düren, Germany). Ultrasound assisted synthesis were carried out employing a Cole-Parmer ultrasonic bath model 8891 (Cole-Parmer, Vernon Hills, IL, USA), operating at $42 \mathrm{kHz}$. Melting points were determined in open capillary tubes with a Büchi M-565 melting point apparatus (Büchi, Flawil, Switzerland) and are uncorrected. Microwave-assisted reactions were carried out in a monomodal reactor (Anton-Paar Monowave 300) equipped with a hydraulic pressure sensing device 
and an infrared temperature-sensor (Anton-Paar, Graz, Austria). ${ }^{1} \mathrm{H}$ NMR and ${ }^{13} \mathrm{C}$ NMR were measured with an Agilent DD2 spectrometer (Agilent, Santa Clara, CA, USA), operating at $600 \mathrm{MHz}$ and $151 \mathrm{MHz}$ for ${ }^{1} \mathrm{H}$ and ${ }^{13} \mathrm{C}$, respectively. Chemical shifts are given in parts per million relatives to tetramethylsilane $\left(\mathrm{Me}_{4} \mathrm{Si}, \delta=0\right)$; $J$ values are given in Hz. Splitting patterns are expressed as follow: s, singlet; $d$, doublet; $t$, triplet; $q$, quartet; dd, doublet of doublet; td, triplet of doublet; ddd, doublet doublet of doublet; dq, doublet of quartets; m, multiplet; bs, broad singlet. High resolution Mass spectra were recorded on a Bruker ESI/APCI-TOF, MicroTOF-II-Focus spectrometer (Bruker, Billerica, MA, USA) by electrospray ionization (ESI); whereas low resolution Mass spectra were recorded on a Waters Xevo TQ-MS spectrometer (Waters, Milford, MA, USA) by electron impact (EI). All compounds were named using the automatic name generator tool from the ChemDraw Professional 16.0.1.4 software (PerkinElmer, Waltham, MA, USA), according to IUPAC rules.

\subsection{Chemistry}

3.2.1. General Procedure for the One-Pot Synthesis of 2-Phenyl-2H-Indazole Derivatives $1-6,9-13,16,17,19$ and 20

A $50 \mathrm{~mL}$ heavy wall glass Schlenk reaction tube was charged with 2-nitrobenzaldehyde $(1.654 \mathrm{mmol})$ and aniline or the substituted aniline $(1.654 \mathrm{mmol})$. The reaction mixture was ultrasonicated for two hours at $40{ }^{\circ} \mathrm{C}$. The condensed water under the tube walls was removed. Afterwards, triethyl phosphite $(5 \mathrm{mmol})$ was added and heated to $150{ }^{\circ} \mathrm{C}$ under nitrogen atmosphere for two hours. The phosphite excess was oxidized with $20 \mathrm{~mL}$ of $5 \%$ hydrogen peroxide solution. The product was extracted with ethyl acetate $(20 \mathrm{~mL} \times 3)$ and the organic layer was washed with brine $(20 \mathrm{~mL})$ and dried with anhydrous sodium sulfate and finally concentrated under vacuum distillation. The evaporation residue was purified by using column chromatography with hexane-ethyl acetate 98:2 (compound 19), 95:5 (compounds, 9-13, 16, 17), 90:10 (compounds 1-3, 6, 20), and 80:20 (compounds 4, 5).

2-Phenyl-2H-Indazole (1), White solid, $92 \%$ yield, m.p.: $81-82{ }^{\circ} \mathrm{C}$. The spectroscopic data agree with the previously reported data $[8,46] .{ }^{1} \mathrm{H}$ NMR $\left(600 \mathrm{MHz}, \mathrm{CDCl}_{3}\right) \delta 8.41(\mathrm{~d}$, $J=0.9 \mathrm{~Hz}, 1 \mathrm{H}), 7.91-789(\mathrm{~m}, 2 \mathrm{H}), 7.79(\mathrm{dd}, J=8.8,0.9 \mathrm{~Hz}, 1 \mathrm{H}), 7.71(\mathrm{dt}, J=8.5,1.0 \mathrm{~Hz}, 1 \mathrm{H})$, 7.54-7.49 (m, 2H), 7.41-7.37 (m, 1H), $7.32(\mathrm{ddd}, J=8.8,6.6,1.0 \mathrm{~Hz}, 1 \mathrm{H}), 7.11$ (ddd, $J=8.4$, 6.6, $0.7 \mathrm{~Hz}, 1 \mathrm{H}) ;{ }^{13} \mathrm{C} \mathrm{NMR}\left(151 \mathrm{MHz}, \mathrm{CDCl}_{3}\right) \delta 149.79,140.54,129.56,127.90,126.83,122.77$, $122.46,121.00,120.41,120.38,117.95$.

2-(4-Chlorophenyl)-2H-Indazole (2), White solid, $62 \%$ yield, m.p.: $143-146{ }^{\circ} \mathrm{C}$. The spectroscopic agree with the previously reported data [8,47]. ${ }^{1} \mathrm{H} \mathrm{NMR}\left(600 \mathrm{MHz}, \mathrm{CDCl}_{3}\right)$ $\delta 8.37(\mathrm{~d}, J=1.0 \mathrm{~Hz}, 1 \mathrm{H}), 7.87-7.83(\mathrm{~m}, 2 \mathrm{H}), 7.77(\mathrm{dq}, J=8.8,0.9 \mathrm{~Hz}, 1 \mathrm{H}), 7.69(\mathrm{dt}, J=8.5$, $1.0 \mathrm{~Hz}, 1 \mathrm{H}), 7.51-7.47(\mathrm{~m}, 2 \mathrm{H}), 7.33(\mathrm{ddd}, J=8.8,6.6,1.1 \mathrm{~Hz}, 1 \mathrm{H}), 7.12(\mathrm{ddd}, J=8.5,6.6$, $0.8 \mathrm{~Hz}, 1 \mathrm{H}) ;{ }^{13} \mathrm{C}$ NMR $\left(151 \mathrm{MHz}, \mathrm{CDCl}_{3}\right) \delta 150.05,139.19,133.72,129.83,127.26,123.03$, $122.87,122.17,120.50,120.42,118.07$.

2-(4-Methoxyphenyl)-2H-Indazole (3), Beige solid, $57 \%$ yield, m.p.: $133-136{ }^{\circ} \mathrm{C}$. The spectroscopic data agree with the previously reported data [8,48]. ${ }^{1} \mathrm{H}$ NMR $(600 \mathrm{MHz}$, $\left.\mathrm{CDCl}_{3}\right) \delta 8.31(\mathrm{~d}, J=0.9 \mathrm{~Hz}, 1 \mathrm{H}), 7.83-7.76(\mathrm{~m}, 3 \mathrm{H}), 7.70(\mathrm{dt}, J=8.4,1.0 \mathrm{~Hz}, 1 \mathrm{H}), 7.32(\mathrm{ddd}$, $J=8.7,6.6,1.0 \mathrm{~Hz}, 1 \mathrm{H}), 7.11(\mathrm{ddd}, J=8.4,6.6,0.8 \mathrm{~Hz}, 1 \mathrm{H}), 7.06-7.00(\mathrm{~m}, 2 \mathrm{H}), 3.86(\mathrm{~s}, 3 \mathrm{H})$; ${ }^{13} \mathrm{C}$ NMR $\left(151 \mathrm{MHz}, \mathrm{CDCl}_{3}\right) \delta 159.42,149.71,134.25,126.66,122.83,122.55,122.35,120.43$, $120.38,117.90,114.77,55.73$.

Methyl 4-(2H-Indazol-2-yl)benzoate (4), White solid, $39 \%$ yield, m.p.: $186-187^{\circ} \mathrm{C}$. The spectroscopic data agree with the previously reported data $[8,48] .{ }^{1} \mathrm{H}$ NMR $(600 \mathrm{MHz}$, $\left.\mathrm{CDCl}_{3}\right) \delta 8.47(\mathrm{~d}, J=0.7 \mathrm{~Hz}, 1 \mathrm{H}), 8.22-8.18(\mathrm{~m}, 2 \mathrm{H}), 8.02-7.99(\mathrm{~m}, 2 \mathrm{H}), 7.77(\mathrm{dd}, J=8.8$, $0.8 \mathrm{~Hz}, 1 \mathrm{H}), 7.69(\mathrm{~d}, J=8.5 \mathrm{~Hz}, 1 \mathrm{H}), 7.33(\mathrm{ddd}, J=8.8,6.6,1.0 \mathrm{~Hz}, 1 \mathrm{H}), 7.14-7.10(\mathrm{~m}, 1 \mathrm{H})$, 3.95 (s, 3H); ${ }^{13} \mathrm{C}$ NMR $\left(151 \mathrm{MHz}, \mathrm{CDCl}_{3}\right) \delta$ 166.32, 150.32, 143.77, 131.30, 129.40, 127.58, $123.14,123.12,120.60,120.39,118.20,52.46$.

2-(4-Fluorophenyl)-2H-Indazole (5), White solid, $71 \%$ yield, m.p.: $110-111{ }^{\circ} \mathrm{C}$. The spectroscopic data agree with the previously reported data [49]. ${ }^{1} \mathrm{H}$ NMR $(600 \mathrm{MHz}$, $\left.\mathrm{CDCl}_{3}\right) \delta 8.34(\mathrm{~d}, J=0.9 \mathrm{~Hz}, 1 \mathrm{H}), 7.88-7.84(\mathrm{~m}, 2 \mathrm{H}), 7.78(\mathrm{dq}, J=8.8,1.0 \mathrm{~Hz}, 1 \mathrm{H}), 7.70(\mathrm{dt}$, $J=8.5,1.0 \mathrm{~Hz}, 1 \mathrm{H}), 7.33(\mathrm{ddd}, J=8.7,6.6,1.0 \mathrm{~Hz}, 1 \mathrm{H}), 7.23-7.19(\mathrm{~m}, 2 \mathrm{H}), 7.12(\mathrm{ddd}, J=8.5$, 
6.6, $0.8 \mathrm{~Hz}, 1 \mathrm{H}) .{ }^{13} \mathrm{C}$ NMR $\left(151 \mathrm{MHz}, \mathrm{CDCl}_{3}\right) \delta 162.05(\mathrm{~d}, J=248.1 \mathrm{~Hz}), 149.83(\mathrm{~s}), 136.88$ $(\mathrm{d}, J=2.7 \mathrm{~Hz}), 126.94(\mathrm{~s}), 122.84(\mathrm{~s}), 122.77(\mathrm{~d}, J=8.6 \mathrm{~Hz}), 122.59(\mathrm{~s}), 120.48(\mathrm{~s}), 120.34(\mathrm{~s})$, $117.89(\mathrm{~s}), 116.45(\mathrm{~d}, J=22.8 \mathrm{~Hz})$.

2-[4-(Trifluoromethyl)phenyl]-2H-Indazole (6), Pale pink solid, 43\% yield, m.p.: 188$190{ }^{\circ} \mathrm{C}$. The spectroscopic data agree with the previously reported data [47]. ${ }^{1} \mathrm{H}$ NMR $\left(600 \mathrm{MHz}, \mathrm{CDCl}_{3}\right) \delta 8.46(\mathrm{~d}, J=0.9 \mathrm{~Hz}, 1 \mathrm{H}), 8.06(\mathrm{~d}, J=8.5 \mathrm{~Hz}, 2 \mathrm{H}), 7.81-7.76(\mathrm{~m}, 3 \mathrm{H}), 7.70$ $(\mathrm{dt}, J=8.5,1.0 \mathrm{~Hz}, 1 \mathrm{H}), 7.34(\mathrm{ddd}, J=8.8,6.6,1.0 \mathrm{~Hz}, 1 \mathrm{H}), 7.13(\mathrm{ddd}, J=8.4,6.6,0.5 \mathrm{~Hz}$, 1H). ${ }^{13} \mathrm{C}$ NMR $\left(600 \mathrm{MHz}, \mathrm{CDCl}_{3}\right) \delta 150.21,142.95,129.75(\mathrm{q}, J=33.1 \mathrm{~Hz}), 127.52,126.86(\mathrm{q}$, $J=3.7 \mathrm{~Hz}), 123.80(\mathrm{q}, J=272.0 \mathrm{~Hz}), 123.08,123.04,120.76,120.47,120.41,118.09$.

2-(3-Chlorophenyl)-2H-Indazole (9), Light pink solid, 53\% yield, m.p.: $109-110{ }^{\circ} \mathrm{C}$. The spectroscopic data agree with the previously reported data $[48,50] .{ }^{1} \mathrm{H}$ NMR $(600 \mathrm{MHz}$, $\left.\mathrm{CDCl}_{3}\right) \delta 8.39(\mathrm{~d}, J=1.0 \mathrm{~Hz}, 1 \mathrm{H}), 7.98(\mathrm{t}, J=2.1 \mathrm{~Hz}, 1 \mathrm{H}), 7.81-7.75(\mathrm{~m}, 2 \mathrm{H}), 7.70(\mathrm{dt}, J=8.5$, $1.0 \mathrm{~Hz}, 1 \mathrm{H}), 7.45(\mathrm{t}, J=8.1 \mathrm{~Hz}, 1 \mathrm{H}), 7.39-7.31(\mathrm{~m}, 2 \mathrm{H}), 7.12(\mathrm{ddd}, J=8.3,6.6,0.8 \mathrm{~Hz}, 1 \mathrm{H})$; ${ }^{13} \mathrm{C}$ NMR $\left(151 \mathrm{MHz}, \mathrm{CDCl}_{3}\right) \delta 149.95,141.45,135.42,130.57,127.88,127.25,122.83,121.24$, $120.41,120.39,118.77,117.99$.

2-(3-Methoxyphenyl)-2H-Indazole (10), Beige solid, $45 \%$ yield, m.p.: $51-52{ }^{\circ} \mathrm{C}$. The spectroscopic data agree with the previously reported data [50]. ${ }^{1} \mathrm{H}$ NMR $(600 \mathrm{MHz}$, $\left.\mathrm{CDCl}_{3}\right) \delta 8.39(\mathrm{~d}, J=1.0 \mathrm{~Hz}, 1 \mathrm{H}), 7.79(\mathrm{dq}, J=8.6,0.8 \mathrm{~Hz}, 1 \mathrm{H}), 7.69(\mathrm{dt}, J=8.5,1.0 \mathrm{~Hz}, 1 \mathrm{H})$, $7.52(\mathrm{t}, J=2.2 \mathrm{~Hz}, 1 \mathrm{H}), 7.44-7.38(\mathrm{~m}, 2 \mathrm{H}), 7.32(\mathrm{ddd}, J=8.8,6.6,1.1 \mathrm{~Hz}, 1 \mathrm{H}), 7.11(\mathrm{ddd}$, $J=8.3,6.5,0.8 \mathrm{~Hz}, 1 \mathrm{H}), 6.93(\mathrm{ddd}, J=7.9,2.5,1.2 \mathrm{~Hz}, 1 \mathrm{H}), 3.90(\mathrm{~s}, 3 \mathrm{H}) ;{ }^{13} \mathrm{C}$ NMR $(151 \mathrm{MHz}$, $\left.\mathrm{CDCl}_{3}\right) \delta 160.58,149.72,141.68,130.27,126.87,122.71,122.48,120.57,120.39,117.94,113.94$, $112.94,106.81,55.61$.

Methyl 3-(2H-Indazol-2-yl)benzoate (11), Beige solid, 51\% yield, m.p.: $103-104{ }^{\circ} \mathrm{C}$. The spectroscopic data agree with the previously reported data [48]. ${ }^{1} \mathrm{H}$ NMR $\left(600 \mathrm{MHz}, \mathrm{CDCl}_{3}\right)$ 8.55-8.53 (m, 1H), $8.48(\mathrm{~d}, J=0.9 \mathrm{~Hz}, 1 \mathrm{H}), 8.18(\mathrm{ddd}, J=8.1,2.3,1.1 \mathrm{~Hz}, 1 \mathrm{H}), 8.08-8.05(\mathrm{~m}$, $1 \mathrm{H}), 7.79(\mathrm{dq}, J=8.8,0.9 \mathrm{~Hz}, 1 \mathrm{H}), 7.71(\mathrm{dt}, J=8.5,1.1 \mathrm{~Hz}, 1 \mathrm{H}), 7.61(\mathrm{t}, J=7.9 \mathrm{~Hz}, 1 \mathrm{H}), 7.33$ (ddd, $J=8.7,6.6,1.0 \mathrm{~Hz}, 1 \mathrm{H}), 7.12(\mathrm{ddd}, J=8.5,6.6,0.9 \mathrm{~Hz}, 1 \mathrm{H}), 3.97(\mathrm{~s}, 3 \mathrm{H}) ;{ }^{13} \mathrm{C} \mathrm{NMR}$ $\left(151 \mathrm{MHz}, \mathrm{CDCl}_{3}\right) \delta 166.10,149.98,140.67,131.69,129.79,128.77,127.18,125.21,122.93$, $122.77,121.51,120.46,120.44,118.00,52.47$.

2-(3-Fluorophenyl)-2H-Indazole (12), White solid, 51\% yield, m.p.: $96-98{ }^{\circ} \mathrm{C}$. The spectroscopic data agree with the previously reported data [48]. ${ }^{1} \mathrm{H} \mathrm{NMR}\left(600 \mathrm{MHz}, \mathrm{CDCl}_{3}\right) \delta$ $8.40(\mathrm{~m}, J=0.8 \mathrm{~Hz}, 1 \mathrm{H}), 7.79-7.76(\mathrm{~m}, 1 \mathrm{H}), 7.73-7.67(\mathrm{~m}, 3 \mathrm{H}), 7.51-7.46(\mathrm{~m}, 1 \mathrm{H}), 7.33(\mathrm{ddd}, J$ $=8.8,6.6,0.9 \mathrm{~Hz}, 1 \mathrm{H}), 7.11(\mathrm{~m}, 2 \mathrm{H}) ;{ }^{13} \mathrm{C} \mathrm{NMR}\left(151 \mathrm{MHz}, \mathrm{CDCl}_{3}\right) \delta 163.22(\mathrm{~d}, J=247.4 \mathrm{~Hz})$, $141.88(\mathrm{~d}, J=10.0 \mathrm{~Hz}), 130.88(\mathrm{~d}, J=9.1 \mathrm{~Hz}), 127.24(\mathrm{~s}), 122.87(\mathrm{~s}), 122.83(\mathrm{~s}), 120.42(\mathrm{~s})$, $120.41(\mathrm{~s}), 118.03(\mathrm{~s}), 116.14(\mathrm{~d}, J=3.1 \mathrm{~Hz}), 114.71(\mathrm{~d}, J=21.2 \mathrm{~Hz}), 108.70(\mathrm{~d}, J=26.2 \mathrm{~Hz})$.

2-[3-(Trifluoromethyl)phenyl]-2H-Indazole (13), White solid, $46 \%$ yield, m.p.: $73-75{ }^{\circ} \mathrm{C}$. The spectroscopic data agree with the previously reported data [48]. ${ }^{1} \mathrm{H}$ NMR $(600 \mathrm{MHz}$, DMSO- $\left.d_{6}\right) \delta 9.29(\mathrm{~d}, J=0.9 \mathrm{~Hz}, 1 \mathrm{H}), 8.48-8.43(\mathrm{~m}, 2 \mathrm{H}), 7.87-7.81(\mathrm{~m}, 2 \mathrm{H}), 7.80(\mathrm{dt}, J=8.5$, $1.0 \mathrm{~Hz}, 1 \mathrm{H}), 7.74(\mathrm{dq}, J=8.8,0.9 \mathrm{~Hz}, 1 \mathrm{H}), 7.36(\mathrm{ddd}, J=8.8,6.5,1.1 \mathrm{~Hz}, 1 \mathrm{H}), 7.14(\mathrm{ddd}$, $J=8.5,6.5,0.8 \mathrm{~Hz}, 1 \mathrm{H}) ;{ }^{13} \mathrm{C}$ NMR $\left(151 \mathrm{MHz}, \mathrm{DMSO}-d_{6}\right) \delta 149.64,140.82,131.55,130.88(\mathrm{q}$, $J=32.9 \mathrm{~Hz}), 127.84,124.69(\mathrm{q}, J=3.8 \mathrm{~Hz}), 124.18(\mathrm{q}, J=272.6 \mathrm{~Hz}), 123.03,122.96,122.72$, $121.52,117.96,117.14(\mathrm{q}, J=3.9 \mathrm{~Hz})$.

2-(2-Chlorophenyl)-2H-Indazole (16), White solid, 55\% yield, m.p.: $60-61^{\circ} \mathrm{C}$. The spectroscopic data agree with the previously reported data [51]. ${ }^{1} \mathrm{H}$ NMR $\left(600 \mathrm{MHz}, \mathrm{CDCl}_{3}\right) \delta$ $8.34(\mathrm{~d}, J=1.0 \mathrm{~Hz}, 1 \mathrm{H}), 7.80(\mathrm{dq}, J=8.6,0.8 \mathrm{~Hz}, 1 \mathrm{H}), 7.74(\mathrm{dt}, J=8.5,1.0 \mathrm{~Hz}, 1 \mathrm{H}), 7.70-7.68$ $(\mathrm{m}, 1 \mathrm{H}), 7.59-7.56(\mathrm{~m}, 1 \mathrm{H}), 7.45-7.40(\mathrm{~m}, 2 \mathrm{H}), 7.35(\mathrm{ddd}, J=8.7,6.6,1.0 \mathrm{~Hz}, 1 \mathrm{H}), 7.14(\mathrm{ddd}$, $J=8.4,6.6,0.7 \mathrm{~Hz}, 1 \mathrm{H}) ;{ }^{13} \mathrm{C} \mathrm{NMR}\left(151 \mathrm{MHz}, \mathrm{CDCl}_{3}\right) \delta 149.40,138.60,130.67,129.94,128.97$, $128.55,127.68,126.92,125.20,122.42,121.99,120.51,117.91$.

2-(2-Methoxyphenyl)-2H-Indazole (17), Light brown oil, 69\% yield. The spectroscopic data agree with the previously reported data [50]. ${ }^{1} \mathrm{H} \mathrm{NMR}\left(600 \mathrm{MHz}, \mathrm{CDCl}_{3}\right) \delta 8.51(\mathrm{~d}$, $J=1.0 \mathrm{~Hz}, 1 \mathrm{H}), 7.87(\mathrm{dd}, J=7.9,1.7 \mathrm{~Hz}, 1 \mathrm{H}), 7.78(\mathrm{dq}, J=8.7,0.9 \mathrm{~Hz}, 1 \mathrm{H}), 7.72(\mathrm{dt}, J=8.4$, $1.0 \mathrm{~Hz}, 1 \mathrm{H}), 7.39(\mathrm{ddd}, J=8.2,7.5,1.7 \mathrm{~Hz}, 1 \mathrm{H}), 7.31(\mathrm{ddd}, J=8.7,6.6,1.1 \mathrm{~Hz}, 1 \mathrm{H}), 7.14-7.07$ $(\mathrm{m}, 3 \mathrm{H}), 3.89(\mathrm{~s}, 3 \mathrm{H}) ;{ }^{13} \mathrm{C}$ NMR $\left(151 \mathrm{MHz}, \mathrm{CDCl}_{3}\right) \delta 151.89,148.82,129.95,129.25,126.63$, $126.52,125.51,122.03,121.84,121.20,120.52,117.71,112.36,56.04$. 
2-(2-Fluorophenyl)-2H-Indazole (19), Yellow oil, 54\% yield. The spectroscopic data agree with the previously reported data $[50,52] .{ }^{1} \mathrm{H} \mathrm{NMR}\left(600 \mathrm{MHz}, \mathrm{CDCl}_{3}\right) \delta 8.50(\mathrm{dd}, J=2.8$, $0.9 \mathrm{~Hz}, 1 \mathrm{H}), 8.08(\mathrm{~m}, 1 \mathrm{H}), 7.79(\mathrm{~m}, 1 \mathrm{H}), 7.72(\mathrm{dt}, J=8.5,0.9 \mathrm{~Hz}, 1 \mathrm{H}), 7.41-7.27(\mathrm{~m}, 4 \mathrm{H}), 7.12$ (ddd, $J=8.5,6.6,0.6 \mathrm{~Hz}, 1 \mathrm{H}) .{ }^{13} \mathrm{C}$ NMR $\left(151 \mathrm{MHz}, \mathrm{CDCl}_{3}\right) \delta 154.20(\mathrm{~d}, J=250.6 \mathrm{~Hz}), 129.12$ $(\mathrm{d}, J=7.9 \mathrm{~Hz}), 125.10(\mathrm{~d}, J=3.7 \mathrm{~Hz}), 124.66(\mathrm{~d}, J=10.0 \mathrm{~Hz}), 122.60(\mathrm{~d}, J=1.3 \mathrm{~Hz}), 117.00(\mathrm{~d}$, $J=20.6 \mathrm{~Hz})$.

2-[2-(Trifluoromethyl)phenyl]-2H-Indazole (20), White solid, 26\% yield, m.p.: 51-52 ${ }^{\circ} \mathrm{C}$. The spectroscopic data agree with the previously reported data [51]. ${ }^{1} \mathrm{H} \mathrm{NMR}(600 \mathrm{MHz}$, $\left.\mathrm{CDCl}_{3}\right) \delta 8.20(\mathrm{~s}, 1 \mathrm{H}), 7.88-7.84(\mathrm{~m}, 1 \mathrm{H}), 7.79(\mathrm{dd}, J=8.8,1.0 \mathrm{~Hz}, 1 \mathrm{H}), 7.75-7.70(\mathrm{~m}, 2 \mathrm{H})$, 7.66-7.62 (m, $J=9.7,4.3 \mathrm{~Hz}, 2 \mathrm{H}), 7.36(\mathrm{ddd}, J=8.8,6.6,1.0 \mathrm{~Hz}, 1 \mathrm{H}), 7.15$ (ddd, $J=8.6,6.6$, $0.8 \mathrm{~Hz}, 1 \mathrm{H}) ;{ }^{13} \mathrm{C}$ NMR $\left(151 \mathrm{MHz}, \mathrm{CDCl}_{3}\right) \delta 149.74,139.11$ (q, $\left.J=1.6 \mathrm{~Hz}\right), 132.83,129.67$, $127.27(q, J=5.1 \mathrm{~Hz}), 127.09,126.42(\mathrm{q}, J=31.7 \mathrm{~Hz}), 125.87(\mathrm{q}, J=1.9 \mathrm{~Hz}), 123.0275(\mathrm{q}, 273.9)$, $122.69,122.24,120.54,118.05,77.16$.

\subsubsection{General Procedure for $O$-Demethylation}

The methoxy-2-phenylindazole compounds (3, 10 or 17) (4 mmol) were dissolved in dichloromethane $(12 \mathrm{~mL})$ and cooled to $0{ }^{\circ} \mathrm{C}$ under $\mathrm{N}_{2}$ atmosphere. Afterwards, boron tribromide (12 mmol, $12 \mathrm{~mL}$ of $1 \mathrm{M}$ solution in dichloromethane) was added and the reaction mixture was stirred at room temperature overnight. The solvent was removed by evaporation and a saturated sodium bicarbonate solution was added. The resulting solid was filtered and dried under vacuum. The crude product was purified using a short column packed with silica gel and ethyl acetate-hexanes (6:4) as a mobile phase.

4-(2H-Indazol-2-yl) phenol (7), White solid, 64\% yield, m.p.: 179-181 ${ }^{\circ} \mathrm{C}$. The spectroscopic data agree with the previously reported data [8]. ${ }^{1} \mathrm{H}$ NMR $\left(600 \mathrm{MHz}, \mathrm{DMSO}-d_{6}\right) \delta$ $9.84(\mathrm{~s}, 1 \mathrm{H}), 8.90(\mathrm{~d}, J=0.9 \mathrm{~Hz}, 1 \mathrm{H}), 7.90-7.83(\mathrm{~m}, 2 \mathrm{H}), 7.74(\mathrm{dt}, J=8.4,1.0 \mathrm{~Hz}, 1 \mathrm{H}), 7.68(\mathrm{dq}$, $J=8.8,0.9 \mathrm{~Hz}, 1 \mathrm{H}), 7.28(\mathrm{ddd}, J=8.7,6.6,1.1 \mathrm{~Hz}, 1 \mathrm{H}), 7.08(\mathrm{ddd}, J=8.3,6.6,0.8 \mathrm{~Hz}, 1 \mathrm{H})$, 6.97-6.92 (m, 2H); ${ }^{13} \mathrm{C}$ NMR (151 MHz, DMSO-d $) \delta 157.18,148.56,132.20,126.19,122.33$, $121.84,121.66,120.87,120.67,117.21,115.90$.

3-(2H-Indazol-2-yl)phenol (14), Beige solid, 47\% yield, m.p.: $131-132{ }^{\circ} \mathrm{C} .{ }^{1} \mathrm{H} \mathrm{NMR}$ $\left(600 \mathrm{MHz}, \mathrm{DMSO}-d_{6}\right) \delta 9.94(\mathrm{~s}, 1 \mathrm{H}), 9.02(\mathrm{~d}, J=0.9 \mathrm{~Hz}, 1 \mathrm{H}), 7.75(\mathrm{dt}, J=8.5,1.0 \mathrm{~Hz}, 1 \mathrm{H})$, $7.70(\mathrm{dq}, J=8.8,0.9 \mathrm{~Hz}, 1 \mathrm{H}), 7.51-7.48(\mathrm{~m}, 2 \mathrm{H}), 7.36(\mathrm{t}, J=8.0 \mathrm{~Hz}, 1 \mathrm{H}), 7.30(\mathrm{ddd}, J=8.8,6.6$, $1.1 \mathrm{~Hz}, 1 \mathrm{H}), 7.09(\mathrm{ddd}, J=8.5,6.5,0.9 \mathrm{~Hz}, 1 \mathrm{H}), 6.84(\mathrm{ddd}, J=8.1,2.4,1.0 \mathrm{~Hz}, 1 \mathrm{H}) ;{ }^{13} \mathrm{C}$ NMR $\left(151 \mathrm{MHz}, \mathrm{DMSO}-d_{6}\right) \delta 158.37,148.77,141.02,130.47,126.70,122.35,122.02,121.51,120.90$, 117.44, 114.84, 110.67, 107.41. MS: $m / z 211.08\left([\mathrm{M}+\mathrm{H}]^{+}, 100 \%\right)$

2-(2H-Indazol-2-yl)phenol (21), White solid, $85 \%$ yield, m.p.: $114-116^{\circ} \mathrm{C}$. The spectroscopic data agree with the previously reported data [52]. ${ }^{1} \mathrm{H} \mathrm{NMR}\left(600 \mathrm{MHz}, \mathrm{CDCl}_{3}\right) \delta$ $12.09(\mathrm{~s}, 1 \mathrm{H}), 8.47(\mathrm{~d}, J=0.8 \mathrm{~Hz}, 1 \mathrm{H}), 7.75-7.71(\mathrm{~m}, 2 \mathrm{H}), 7.58(\mathrm{dd}, J=8.1,1.5 \mathrm{~Hz}, 1 \mathrm{H}), 7.38$ (ddd, $J=8.7,6.6,1.0 \mathrm{~Hz}, 1 \mathrm{H}), 7.29-7.25(\mathrm{~m}, 1 \mathrm{H}), 7.19-7.15(\mathrm{~m}, 2 \mathrm{H}), 6.97$ (ddd, $J=8.2,7.3$, $1.4 \mathrm{~Hz}, 1 \mathrm{H}) ;{ }^{13} \mathrm{C} \mathrm{NMR}\left(151 \mathrm{MHz}, \mathrm{CDCl}_{3}\right) \delta 150.48,147.61,129.08,127.69,124.84,122.96$, $121.32,120.28,120.21,119.63,119.43,119.22,116.82$.

\subsubsection{General Procedure for the Hydrolysis of Ester Derivatives}

The methyl ester compounds ( 4 or 11, $1.2 \mathrm{mmol}$ ) were dissolved in methanol $(7.5 \mathrm{~mL})$ and an aqueous solution of $\mathrm{NaOH}(3.6 \mathrm{mmol}$ in $3 \mathrm{~mL}$ of water) was added. The reaction mixture was refluxed until the starting material was totally consumed. The mixture was cooled on ice and acidified to $\mathrm{pH} 1$ with $\mathrm{HCl}$ to induce precipitation. The solid was filtered under vacuum and dried.

4-(2H-Indazol-2-yl)benzoic Acid (8), White solid, 96\% yield, m.p.: $288-289{ }^{\circ} \mathrm{C}$. The spectroscopic data agree with the previously reported data [8]. ${ }^{1} \mathrm{H}$ NMR $(600 \mathrm{MHz}, \mathrm{DMSO}-$ $\left.d_{6}\right) \delta 9.21(\mathrm{~s}, 1 \mathrm{H}), 8.26-8.23(\mathrm{~m}, 2 \mathrm{H}), 8.15-8.11(\mathrm{~m}, 2 \mathrm{H}), 7.78(\mathrm{dt}, J=8.5,1.0 \mathrm{~Hz}, 1 \mathrm{H}), 7.72$ $(\mathrm{dd}, J=8.8,0.9 \mathrm{~Hz}, 2 \mathrm{H}), 7.33(\mathrm{ddd}, J=8.8,6.5,1.1 \mathrm{~Hz}, 1 \mathrm{H}), 7.12(\mathrm{ddd}, J=8.5,6.6,0.8 \mathrm{~Hz}$, $1 \mathrm{H}) ;{ }^{13} \mathrm{C}$ NMR $(151 \mathrm{MHz}$, DMSO-d 6 ) $\delta 166.54,149.30,142.91,130.90,129.74,127.36,122.62$, $122.52,122.13,121.07,119.94,117.57$. 
3-(2H-Indazol-2-yl)benzoic Acid (15), White solid, 43\% yield, m.p.: 215-217 ${ }^{\circ} \mathrm{C} .1 \mathrm{H} \mathrm{NMR}$ $\left(600 \mathrm{MHz}\right.$, acetone- $\left.d_{6}\right) \delta 9.01(\mathrm{~s}, 1 \mathrm{H}), 8.78-8.69(\mathrm{~m}, 1 \mathrm{H}), 8.36(\mathrm{ddd}, J=8.1,2.3,1.0 \mathrm{~Hz}, 1 \mathrm{H})$, 8.12-8.05 (m, 1H), 7.82-7.69 (m, $J=11.5,9.0,4.6 \mathrm{~Hz}, 3 \mathrm{H}), 7.33(\mathrm{ddd}, J=8.7,6.6,0.9 \mathrm{~Hz}, 1 \mathrm{H})$, 7.15-7.08 (m, 1H) ${ }^{13} \mathrm{C}$ NMR (151 MHz, acetone- $\left.d_{6}\right) \delta 166.84,150.61,141.64,133.09,130.81$, $129.46,127.85,125.33,123.98,123.28,122.20,122.05,121.73,118.63$. MS: $m / z 239.13$ ([M + $\left.\mathrm{H}]^{+}, 100 \%\right)$.

\subsubsection{Synthesis of Compound 18 and 22}

2-(2H-Indazol-2-yl)benzonitrile (18a), Synthesized by a slight variation of the general method (Section 3.2.1). Briefly, the reaction mixture was ultrasonicated by four cycles of $1 \mathrm{~h}$ at $40^{\circ} \mathrm{C}$; after each $1 \mathrm{~h}$ sonication step, the mixture was maintained under vacuum for $30 \mathrm{~min}$ at $30 \mathrm{mmHg}$ and $40^{\circ} \mathrm{C}$. The cyclization with triethyl phosphite and the reaction workup were carried out as previously described (vide supra). The crude product was purified by using column chromatography with hexane-ethyl acetate 90:10. White solid, $32 \%$ yield, m.p.: $128-129^{\circ} \mathrm{C}$. The spectroscopic data agree with the previously reported data [50]. ${ }^{1} \mathrm{H}$ NMR $\left(600 \mathrm{MHz} \mathrm{CDCl}_{3}\right) \delta 8.60(\mathrm{~d}, J=0.9 \mathrm{~Hz}, 1 \mathrm{H}), 7.97(\mathrm{dd}, J=8.2,1.1 \mathrm{~Hz}$, $1 \mathrm{H}), 7.85(\mathrm{dd}, J=7.8,1.4 \mathrm{~Hz}, 1 \mathrm{H}), 7.82-7.76(\mathrm{~m}, 2 \mathrm{H}), 7.54(\mathrm{td}, J=7.7,1.1 \mathrm{~Hz}, 1 \mathrm{H}), 7.36(\mathrm{ddd}$, $J=8.8,6.6,1.1 \mathrm{~Hz}, 1 \mathrm{H}), 7.15(\mathrm{ddd}, J=8.5,6.6,0.8 \mathrm{~Hz}, 1 \mathrm{H}) ;{ }^{13} \mathrm{C} \mathrm{NMR}\left(151 \mathrm{MHz}, \mathrm{CDCl}_{3}\right) \delta$ $150.22,142.36,134.52,134.04,128.45,127.68,125.98,123.42,123.09,122.84,120.70,118.03$, $116.55,106.82$.

2-(2H-Indazol-2-yl)benzoic Acid (22), A $50 \mathrm{~mL}$ round bottom flask was charged with 2-(2H-indazol-2-yl)benzonitrile $(2 \mathrm{mmol})$, sodium hydroxide $(16 \mathrm{mmol})$ and water $(25 \mathrm{~mL})$. The mixture was heated to reflux for four hours. The cold mixture was treated with aqueous solution of $\mathrm{HCl}(10 \%)$ till total precipitation, the solid was filtered under vacuum and washed with cold water. White solid, 90\% yield, m.p.: $210-211{ }^{\circ} \mathrm{C} .{ }^{1} \mathrm{H}$ NMR $(600$ $\left.\mathrm{MHz}, \mathrm{DMSO}-\mathrm{d}_{6}\right) \delta 12.97(\mathrm{~s}, 1 \mathrm{H}), 8.72(\mathrm{~d}, J=0.9 \mathrm{~Hz}, 1 \mathrm{H}), 7.86-7.83(\mathrm{~m}, 1 \mathrm{H}), 7.78(\mathrm{dt}, J=8.5$, $1.0 \mathrm{~Hz}, 1 \mathrm{H}), 7.75-7.71(\mathrm{~m}, 2 \mathrm{H}), 7.66(\mathrm{dd}, J=8.8,0.9 \mathrm{~Hz}, 1 \mathrm{H}), 7.63(\mathrm{ddd}, J=7.7,6.3,2.4 \mathrm{~Hz}$, 1H), 7.33-7.28 (m, 1H), 7.13-7.09 (m, 1H); ${ }^{13} \mathrm{C} \mathrm{NMR} \mathrm{(151} \mathrm{MHz,} \mathrm{DMSO-d} 6$ ) $\delta 167.36,148.77$, $138.88,131.78,129.83,129.53,128.92,126.32,126.26,124.86,122.07,121.69,120.97,117.38$. MS (HR-ESI) for $\mathrm{C}_{14} \mathrm{H}_{11} \mathrm{~N}_{2} \mathrm{O}_{2}[\mathrm{M}+\mathrm{H}]^{+}$, calcd: $m / z 239.0815$, found: $m / z 239.0824$.

Methyl 2-(2H-Indazol-2-yl)benzoate (18), A $10 \mathrm{~mL}$ microwave vial was charged with 2-(2H-indazol-2-yl)benzoic acid $(1.26 \mathrm{mmol})$ and $5 \mathrm{~mL}$ of solution of $\mathrm{HCl}$ in $\mathrm{MeOH}(15 \%)$. The reaction was heated at $120{ }^{\circ} \mathrm{C}$ under microwave irradiation for $25 \mathrm{~min}$. Then the reaction was neutralized with $10 \%$ solution of $\mathrm{NaHCO}_{3}$ and extracted with dichloromethane $(3 \times 10 \mathrm{~mL})$. The combined organic layers were dried over $\mathrm{Na}_{2} \mathrm{SO}_{4}$ and concentrated under vacuum. The product was purified by column chromatography employing hexanesdichloromethane-ethyl acetate (50:35:15) as mobile phase. White solid, 36\% yield, m.p.: $71-73{ }^{\circ} \mathrm{C}$. The spectroscopic data agree with the previously reported data [48]. ${ }^{1} \mathrm{H}$ NMR $\left(600 \mathrm{MHz}_{\mathrm{CDCl}}\right) \delta 8.21(\mathrm{~d}, J=0.9 \mathrm{~Hz}, 1 \mathrm{H}), 7.90(\mathrm{dd}, J=7.7,1.4 \mathrm{~Hz}, 1 \mathrm{H}), 7.76(\mathrm{dd}, J=8.8$, $0.9 \mathrm{~Hz}, 1 \mathrm{H}), 7.72(\mathrm{dt}, J=8.5,0.8 \mathrm{~Hz}, 1 \mathrm{H}), 7.66-7.59(\mathrm{~m}, J=9.2,7.9,1.4 \mathrm{~Hz}, 2 \mathrm{H}), 7.54(\mathrm{td}$, $J=7.6,1.4 \mathrm{~Hz}, 1 \mathrm{H}), 7.32(\mathrm{ddd}, J=8.8,6.6,1.0 \mathrm{~Hz}, 1 \mathrm{H}), 7.12(\mathrm{ddd}, J=8.4,6.6,0.6 \mathrm{~Hz}, 1 \mathrm{H})$, $3.61(\mathrm{~s}, 3 \mathrm{H}) ;{ }^{13} \mathrm{C}$ NMR $\left(151 \mathrm{MHz} \mathrm{CDCl}_{3}\right) \delta 166.81,149.70,139.80,132.07,130.56,128.82$, $128.33,126.66,126.33,123.81,122.36,122.34,120.41,117.93,52.46$.

\subsection{Antiprotozoal Activity Assays}

Trichomonas vaginalis strain GT3, Giardia intestinalis isolate IMSS:0981:1 and Entamoeba histolytica strain HM1-IMSS were used in all the experiments. Trophozoites of G. intestinalis were maintained in a TYI-S-33 medium supplemented with $10 \%$ calf serum and bovine bile. E. histolytica and T. vaginalis trophozoites were maintained in TYI-S-33 medium supplemented with $10 \%$ bovine serum. Briefly, $5 \times 10^{4}$ trophozoites of G. intestinalis or T. vaginalis, or $6 \times 10^{3}$ trophozoites of E. histolytica were incubated for $48 \mathrm{~h}$ at $37^{\circ} \mathrm{C}$ with different concentrations of the compound to be tested, each added as solutions in DMSO. As a negative control, parasites received an equivalent amount of DMSO only, while albendazole (ABZ) and metronidazole (MTZ) were included as positive controls. At 
the end of the treatment period, the cells were washed and subcultured for another $48 \mathrm{~h}$ in fresh medium to which no drug was added. The trophozoites were then counted with a hemocytometer and the $50 \%$ inhibitory concentration $\left(\mathrm{IC}_{50}\right)$, together with the respective 95\% confidence limit was calculated by Probit analysis. Experiments were carried out in triplicate and repeated at least twice.

\subsection{Cheminformatic Studies}

\subsubsection{Molecular Databases}

Compounds with $\mathrm{IC}_{50}$ annotations against E. histolytica, G. intestinalis and T. vaginalis were retrieved from ChEMBL version 27 [37]. The crude databases were refined by using the following criteria: (a) compounds having structural errors or not readable were eliminated; (b) duplicated compounds were removed to preserve a single structure (the lowest activity value was kept); (c) only compounds with values of $\mathrm{IC}_{50}<100 \mu \mathrm{M}$ were considered; d) counterions were removed from salts and charges were neutralized; (e) inorganic compounds were removed. The curated databases contain 968 compounds against $E$. histolytica, 1211 against $G$. intestinalis and 445 against $T$. vaginalis with $\mathrm{pIC}_{50}$ annotations $\left(-\log \mathrm{IC}_{50}[\mathrm{~mol} / \mathrm{L}]\right)$. Moreover, 180 benzimidazole derivatives with activity against these same protozoa were taken from previous reports to give 111 derivatives with $\mathrm{IC}_{50}$ against $E$. histolytica, 159 compounds against $G$. intestinalis and 134 molecules against $T$. vaginalis $[7,23-36]$. Compounds were drawing employing ChemDraw Professional 16.0.1.4 software and exported as simplified molecular-input line-entry system (SMILES) with their $\mathrm{pIC}_{50}$ annotations. Moreover, indazole derivatives (1-22) were processed employing the same methodology.

\subsubsection{Property Space}

A set of six properties were calculated for all compounds under study employing RDKit 2019.09.3 implemented in python 3.7.6: Molecular Weight (MW), Rotatable bonds (RBs), Hydrogen Bond Acceptors (HBAs), Hydrogen Bond Donors (HBDs), Topological Polar Surface Area (TPSA), and Octanol-Water Partition Coefficient (logP). Principal Component Analysis (PCA) were carried out employing the OSIRIS DataWarrior software Version 5.2.1 [41]. Violin plots were calculated employing the Seaborn 0.11.1 library implemented in python 3.7.6.

\subsubsection{Structure-Activity Similarity (SAS) Maps}

SAS maps were calculated by employing the Activity Landscape Plotter V1 [53]. Molecular ACCess System (MACCS) keys fingerprint (166 bits) and Extended Connectivity Fingerprint (ECFP) with a diameter four were used $[54,55]$. Each map was generated by plotting molecular similarity in the $X$-axis and the absolute value of the activity differences in the $Y$-axis. The Tanimoto coefficient was used to calculate $N(N-1) / 2$ pairwise structural similarities $\left(\mathrm{SS}_{i, j}\right)$ for each pair of molecules $i$ and $j$ included in each scaffold class (benzimidazole or indazole). Moreover, $N(N-1) / 2$ pairwise activity differences, determined by the equation $\left|\Delta \mathrm{A}(\mathrm{T})_{i, j}\right|=\left|\mathrm{A}(\mathrm{T})_{i}-\mathrm{A}(\mathrm{T})_{j}\right|$, were calculated for indazole and benzimidazole datasets; where $\mathrm{A}(\mathrm{T})_{i}$ and $\mathrm{A}(\mathrm{T})_{j}$ are the activities of the $i$ th and $j$ th molecules $(j>i)$, in $\mathrm{pIC}_{50}$, tested against the target parasite $\mathrm{T}$ [42]. SAS maps were divided in four regions employing a threshold of two units on activity difference (100-fold in potency) and the median similarity of the total datapoints plotted in each map (including indazole and benzimidazole derivatives). Briefly, Regions I and II are associated with scaffold hopping (or side chain hopping) and smooth SAR, respectively, whereas Region IV indicates discontinuous SAR (Figure 4).

\section{Conclusions}

A one-pot procedure including a combination of ultrasound synthesis under neat conditions and a Cadogan's cyclization was implemented for the synthesis of 2-phenyl-2Hindazole derivatives. The one-pot method leads to similar or better yields as compared 
to our previous reports. The biological assays revealed that electron withdrawing groups at the 2-phenyl ring are favorable for antiprotozoal activity. Furthermore, although the SAR information obtained from indazole derivatives as antiprotozoals is still limited, cheminformatic analysis for the 22 compounds studied, highlight their potent values and their smooth SAR nature as compared to reference databases. More studies are needed to expand the activity landscape of indazole derivatives; however, these results represent a valuable start-point toward the optimization of indazole derivatives as antiprotozoals.

Supplementary Materials: The following are available online, Figures S1-S23 ${ }^{1} \mathrm{H}$ NMR and ${ }^{13} \mathrm{C}$ NMR for 2-phenyl-2H-indazole derivatives synthesized (1-22 and 18a).

Author Contributions: Conceptualization, K.R.-V., L.Y.-M., and J.P.-V. methodology, K.R.-V., J.P.-V., L.Y.-M., O.S.-A., J.F.P.-E., and F.C.-B.; formal analysis, J.P.-V., and K.S.O.D.A.; investigation, K.R.V., J.P.-V., M.C.-G., J.D.A.-P., E.A.Q.-S., and K.S.O.D.A.; data curation, K.S.O.D.A.; writing-J.P.-V.; writing-review and editing, L.Y.-M., O.S.-A., J.F.P.-E., and F.C.-B.; supervision, J.P.-V., J.F.P.-E., F.C.-B., and O.S.-A.; project administration, J.P.-V.; funding acquisition, J.P.-V. All authors have read and agreed to the published version of the manuscript.

Funding: This research was funded by Consejo Nacional de Ciencia y Tecnología (CONACyT), grant numbers CB-2015-01-258554 and 1238.

Data Availability Statement: The data presented in this study are available on request from the corresponding author.

Acknowledgments: The authors would like to express their sincere thanks to Amparo Tapia for the technical support with biological assays as well as Ernesto Sánchez Mendoza and Mónica A. Rincón for their analytical support. Moreover, the authors acknowledge to DIFACQUIM for providing the web server to aid the cheminformatic study, OpenMolecules.org for providing the DataWarrior software and Pharmometrica research center for the support with mass spectra determinations.

Conflicts of Interest: The authors declare no conflict of interest.

Sample Availability: Samples of the compounds 1-22 and 18a are available from the authors.

\section{References}

1. Scott, L.J. Niraparib: First global approval. Drugs 2017, 77, 1029-1034. [CrossRef]

2. Al-Salama, Z.T.; Keam, S.J. Entrectinib: First global approval. Drugs 2019, 79, 1477-1483. [CrossRef] [PubMed]

3. Thangadurai, A.; Minu, M.; Wakode, S.; Agrawal, S.; Narasimhan, B. Indazole: A medicinally important heterocyclic moiety. Med. Chem. Res. 2012, 21, 1509-1523. [CrossRef]

4. Hugo, C.; Alejandra, G.; Mercedes, G.; Vicente, J.A.; Carmen, O.d.O. Pharmacological properties of indazole derivatives: Recent developments. Mini. Rev. Med. Chem. 2005, 5, 869-878.

5. Denya, I.; Malan, S.F.; Joubert, J. Indazole derivatives and their therapeutic applications: A patent review (2013-2017). Expert. Opin. Ther. Pat. 2018, 28, 441-453. [CrossRef]

6. Zhang, S.-G.; Liang, C.-G.; Zhang, W.-H. Recent advances in indazole-containing derivatives: Synthesis and biological perspectives. Molecules 2018, 23, 2783. [CrossRef]

7. López-Vallejo, F.; Castillo, R.; Yépez-Mulia, L.; Medina-Franco, J.L. Benzotriazoles and indazoles are scaffolds with biological activity against Entamoeba histolytica. J. Biomol. Screen. 2011, 16, 862-868. [CrossRef]

8. Pérez-Villanueva, J.; Yépez-Mulia, L.; González-Sánchez, I.; Palacios-Espinosa, J.F.; Soria-Arteche, O.; Sainz-Espuñes, T.D.R.; Cerbón, M.A.; Rodríguez-Villar, K.; Rodríguez-Vicente, A.K.; Cortés-Gines, M.; et al. synthesis and biological evaluation of $2 \mathrm{H}$-indazole derivatives: Towards antimicrobial and anti-inflammatory dual agents. Molecules 2017, 22, 1864. [CrossRef]

9. Marrero-Ponce, Y.; Meneses-Marcel, A.; Castillo-Garit, J.A.; Machado-Tugores, Y.; Escario, J.A.; Barrio, A.G.; Pereira, D.M.; Nogal-Ruiz, J.J.; Arán, V.J.; Martínez-Fernández, A.R.; et al. Predicting antitrichomonal activity: A computational screening using atom-based bilinear indices and experimental proofs. Bioorg. Med. Chem. 2006, 14, 6502-6524. [CrossRef]

10. Kantor, M.; Abrantes, A.; Estevez, A.; Schiller, A.; Torrent, J.; Gascon, J.; Hernandez, R.; Ochner, C. Entamoeba histolytica: Updates in clinical manifestation, pathogenesis, and vaccine development. Can. J. Gastroenterol. Hepatol. 2018, 2018, 4601420. [CrossRef]

11. Cacciò, S.; Sprong, H. Epidemiology of giardiasis in humans. In Giardia a Model Organism, 1st ed.; Lujan, H.D., Svärd, S., Eds.; Springer: Vienna, Austria, 2011; Volume 1, pp. 17-28.

12. Hodges, K.; Gill, R. Infectious diarrhea: Cellular and molecular mechanisms. Gut. Microb. 2010, 1, 4-21. [CrossRef]

13. Bala, V.; Chhonker, Y.S. Recent developments in anti-Trichomonas research: An update review. Eur. J. Med. Chem. 2018, 143, 232-243. [CrossRef] 
14. Rowley, J.; Vander Hoorn, S.; Korenromp, E.; Low, N.; Unemo, M.; Abu-Raddad, L.J.; Chico, R.M.; Smolak, A.; Newman, L.; Gottlieb, S.; et al. Chlamydia, gonorrhoea, trichomoniasis and syphilis: Global prevalence and incidence estimates, 2016. Bull. World Health Organ. 2019, 97, 548P-562P. [CrossRef]

15. Schwebke, J.R. Update of trichomoniasis. Sex. Transm. Infect. 2002, 78, 378-379. [CrossRef] [PubMed]

16. Upcroft, P.; Upcroft, J.A. Drug targets and mechanisms of resistance in the anaerobic protozoa. Clin. Microbiol. Rev. 2001, 14, 150-164. [CrossRef] [PubMed]

17. Upcroft, J.A.; Upcroft, P. Drug susceptibility testing of anaerobic protozoa. Antimicrob. Agents Chemother. 2001, 45, 1810-1814. [CrossRef]

18. García, C.G.; Marchat, L.A.; López-Cánovas, L.; Ishiwara, D.G.P.; Rodríguez, M.A.; Orozco, E. Drug resistance mechanisms in Entamoeba histolytica, Giardia lamblia, Trichomonas vaginalis, and opportunistic anaerobic protozoa. In Antimicrobial Drug Resistance: Mechanisms of Drug Resistance, 2nd ed.; Mayers, D.L., Sobel, J.D., Ouellette, M., Kaye, K.S., Marchaim, D., Eds.; Springer International Publishing: Cham, Switzerland, 2017; Volume 1, pp. 613-628.

19. Leitsch, D. Drug resistance in the microaerophilic parasite Giardia lamblia. Curr. Trop. Med. Rep. 2015, 2, 128-135. [CrossRef] [PubMed]

20. Crawford, D.E. Solvent-free sonochemistry: Sonochemical organic synthesis in the absence of a liquid medium. Beilstein J. Org. Chem. 2017, 13, 1850-1856. [CrossRef] [PubMed]

21. Cadogan, J.; Mackie, R. 2-Phenylindazole. Org. Synth. 1968, 48, 113.

22. Schaeffer, L. The role of functional groups in drug-receptor interactions. In The Practice of Medicinal Chemistry, 4th ed.; Wermuth, C.G., Aldous, D., Raboisson, P., Rognan, D., Eds.; Academic Press: San Diego, CA, USA, 2008; Volume 1, pp. 359-378.

23. Navarrete-Vazquez, G.; Rojano-Vilchis, M.D.; Yepez-Mulia, L.; Melendez, V.; Gerena, L.; Hernandez-Campos, A.; Castillo, R.; Hernandez-Luis, F. Synthesis and antiprotozoal activity of some 2-(trifluoromethyl)-1H-benzimidazole bioisosteres. Eur. J. Med. Chem. 2006, 41, 135-141. [CrossRef]

24. Navarrete-Vazquez, G.; Yepez, L.; Hernandez-Campos, A.; Tapia, A.; Hernandez-Luis, F.; Cedillo, R.; Gonzalez, J.; MartinezFernandez, A.; Martinez-Grueiro, M.; Castillo, R. Synthesis and antiparasitic activity of albendazole and mebendazole analogues. Bioorg. Med. Chem. 2003, 11, 4615-4622. [CrossRef]

25. Andrzejewska, M.; Yepez-Mulia, L.; Cedillo-Rivera, R.; Tapia, A.; Vilpo, L.; Vilpo, J.; Kazimierczuk, Z. Synthesis, antiprotozoal and anticancer activity of substituted 2-trifluoromethyl- and 2-pentafluoroethylbenzimidazoles. Eur. J. Med. Chem. 2002, 37, 973-978. [CrossRef]

26. Andrzejewska, M.; Yepez-Mulia, L.; Tapia, A.; Cedillo-Rivera, R.; Laudy, A.E.; Starosciak, B.J.; Kazimierczuk, Z. Synthesis, and antiprotozoal and antibacterial activities of S-substituted 4,6-dibromo- and 4,6-dichloro-2-mercaptobenzimidazoles. Eur. J. Pharm. Sci. 2004, 21, 323-329. [CrossRef] [PubMed]

27. Valdez-Padilla, D.; Rodriguez-Morales, S.; Hernandez-Campos, A.; Hernandez-Luis, F.; Yepez-Mulia, L.; Tapia-Contreras, A.; Castillo, R. Synthesis and antiprotozoal activity of novel 1-methylbenzimidazole derivatives. Bioorg. Med. Chem. 2009, 17, 1724-1730. [CrossRef] [PubMed]

28. Navarrete-Vazquez, G.; Cedillo, R.; Hernandez-Campos, A.; Yepez, L.; Hernandez-Luis, F.; Valdez, J.; Morales, R.; Cortes, R.; Hernandez, M.; Castillo, R. Synthesis and antiparasitic activity of 2-(trifluoromethyl)-benzimidazole derivatives. Bioorg. Med. Chem. Lett. 2001, 11, 187-190. [CrossRef]

29. Hernandez-Luis, F.; Hernandez-Campos, A.; Castillo, R.; Navarrete-Vazquez, G.; Soria-Arteche, O.; Hernandez-Hernandez, M.; Yepez-Mulia, L. Synthesis and biological activity of 2-(trifluoromethyl)- $1 H$-benzimidazole derivatives against some protozoa and Trichinella Spiralis. Eur. J. Med. Chem. 2010, 45, 3135-3141. [CrossRef]

30. Valdez, J.; Cedillo, R.; Hernandez-Campos, A.; Yepez, L.; Hernandez-Luis, F.; Navarrete-Vazquez, G.; Tapia, A.; Cortes, R.; Hernandez, M.; Castillo, R. Synthesis and antiparasitic activity of $1 H$-benzimidazole derivatives. Bioorg. Med. Chem. Lett. 2002, 12, 2221-2224. [CrossRef]

31. Pérez-Villanueva, J.; Medina-Franco, J.L.; Caulfield, T.R.; Hernández-Campos, A.; Hernández-Luis, F.; Yépez-Mulia, L.; Castillo, R. Comparative molecular field analysis (CoMFA) and comparative molecular similarity indices analysis (CoMSIA) of some benzimidazole derivatives with trichomonicidal activity. Eur. J. Med. Chem. 2011, 46, 3499-3508. [CrossRef]

32. Pérez-Villanueva, J.; Hernández-Campos, A.; Yépez-Mulia, L.; Méndez-Cuesta, C.; Méndez-Lucio, O.; Hernández-Luis, F.; Castillo, R. Synthesis and antiprotozoal activity of novel 2-\{[2-(1H-imidazol-1-yl)ethyl]sulfanyl $\}-1 H$-benzimidazole derivatives. Bioorg. Med. Chem. Lett. 2013, 23, 4221-4224. [CrossRef]

33. Soria-Arteche, O.; Hernández-Campos, A.; Yépez-Mulia, L.; Trejo-Soto, P.J.; Hernández-Luis, F.; Gres-Molina, J.; Maldonado, L.A.; Castillo, R. Synthesis and antiprotozoal activity of nitazoxanide-N-methylbenzimidazole hybrids. Bioorg. Med. Chem. Lett. 2013, 23, 6838-6841. [CrossRef]

34. Lopez-Vallejo, F.; Medina-Franco, J.L.; Hernandez-Campos, A.; Rodriguez-Morales, S.; Yepez, L.; Cedillo, R.; Castillo, R. Molecular modeling of some $1 \mathrm{H}$-benzimidazole derivatives with biological activity against Entamoeba Histolytica: A comparative molecular field analysis study. Bioorg. Med. Chem. 2007, 15, 1117-1126. [CrossRef] [PubMed]

35. Hernández-Covarrubias, C.; Vilchis-Reyes, M.A.; Yépez-Mulia, L.; Sánchez-Díaz, R.; Navarrete-Vázquez, G.; Hernández-Campos, A.; Castillo, R.; Hernández-Luis, F. Exploring the interplay of physicochemical properties, membrane permeability and giardicidal activity of some benzimidazole derivatives. Eur. J. Med. Chem. 2012, 52, 193-204. [CrossRef] [PubMed] 
36. Flores-Carrillo, P.; Velázquez-López, J.M.; Aguayo-Ortiz, R.; Hernández-Campos, A.; Trejo-Soto, P.J.; Yépez-Mulia, L.; Castillo, R. Synthesis, antiprotozoal activity, and chemoinformatic analysis of 2-(methylthio)- $1 H$-benzimidazole-5-carboxamide derivatives: Identification of new selective giardicidal and trichomonicidal compounds. Eur. J. Med. Chem. 2017, 137, 211-220. [CrossRef] [PubMed]

37. Gaulton, A.; Bellis, L.J.; Bento, A.P.; Chambers, J.; Davies, M.; Hersey, A.; Light, Y.; McGlinchey, S.; Michalovich, D.; Al-Lazikani, B.; et al. ChEMBL: A large-scale bioactivity database for drug discovery. Nucleic Acids Res. 2011, 40, D1100-D1107. [CrossRef]

38. Pérez-Villanueva, J.; Santos, R.; Hernández-Campos, A.; Giulianotti, M.A.; Castillo, R.; Medina-Franco, J.L. Towards a systematic characterization of the antiprotozoal activity landscape of benzimidazole derivatives. Bioorg. Med. Chem. 2010, 18, 7380-7391. [CrossRef]

39. Pérez-Villanueva, J.; Santos, R.; Hernández-Campos, A.; Giulianotti, M.A.; Castillo, R.; Medina-Franco, J.L. Structure-activity relationships of benzimidazole derivatives as antiparasitic agents: Dual activity-difference (DAD) maps. MedChemComm 2011, 2, 44-49. [CrossRef]

40. Aguayo-Ortiz, R.; Pérez-Villanueva, J.; Hernández-Campos, A.; Castillo, R.; Meurice, N.; Medina-Franco, J.L. Chemoinformatic characterization of activity and selectivity switches of antiprotozoal compounds. Future Med. Chem. 2014, 6, 281-294. [CrossRef]

41. Sander, T.; Freyss, J.; von Korff, M.; Rufener, C. DataWarrior: An open-source program for chemistry aware data visualization and analysis. J. Chem. Inf. Model. 2015, 55, 460-473. [CrossRef] [PubMed]

42. Pérez-Villanueva, J.; Méndez-Lucio, O.; Soria-Arteche, O.; Medina-Franco, J.L. Activity cliffs and activity cliff generators based on chemotype-related activity landscapes. Mol. Divers. 2015, 19, 1021-1035. [CrossRef]

43. Maggiora, G.M. On outliers and activity cliffs - Why QSAR often disappoints. J. Chem. Inf. Model. 2006, 46, 1535. [CrossRef]

44. Bajorath, J.; Peltason, L.; Wawer, M.; Guha, R.; Lajiness, M.S.; Van Drie, J.H. Navigating structure-activity landscapes. Drug Discov. Today 2009, 14, 698-705. [CrossRef]

45. Cruz-Monteagudo, M.; Medina-Franco, J.L.; Pérez-Castillo, Y.; Nicolotti, O.; Cordeiro, M.N.D.S.; Borges, F. Activity cliffs in drug discovery: Dr Jekyll or Mr Hyde? Drug Discov. Today 2014, 19, 1069-1080. [CrossRef]

46. Fang, Y.; Wu, C.; Larock, R.C.; Shi, F. Synthesis of $2 H$-indazoles by the [3 + 2] dipolar cycloaddition of sydnones with arynes. $J$. Org. Chem. 2011, 76, 8840-8851. [CrossRef]

47. Kumar, M.R.; Park, A.; Park, N.; Lee, S. Consecutive condensation, C-N and N-N bond formations: A copper- catalyzed one-pot three-component synthesis of $2 H$-indazole. Org. Lett. 2011, 13, 3542-3545. [CrossRef]

48. Genung, N.E.; Wei, L.; Aspnes, G.E. Regioselective Synthesis of $2 H$-indazoles using a mild, one-pot condensation-Cadogan reductive cyclization. Org. Lett. 2014, 16, 3114-3117. [CrossRef] [PubMed]

49. Elie, J.; Vercouillie, J.; Arlicot, N.; Lemaire, L.; Bidault, R.; Bodard, S.; Hosselet, C.; Deloye, J.-B.; Chalon, S.; Emond, P.; et al. Design of selective COX-2 inhibitors in the (aza)indazole series. Chemistry, in vitro studies, radiochemistry and evaluations in rats of a $\left[{ }^{18} \mathrm{~F}\right]$ PET tracer. J. Enzyme Inhib. Med. Chem. 2019, 34, 1-7. [CrossRef]

50. Schoene, J.; Bel Abed, H.; Schmieder, P.; Christmann, M.; Nazaré, M. A general one-pot synthesis of $2 H$-indazoles using an organophosphorus-silane system. Chem. Eur. J. 2018, 24, 9090-9100. [CrossRef] [PubMed]

51. Zhang, R.; Liu, Z.; Peng, Q.; Zhou, Y.; Xu, L.; Pan, X. Access to 2-substituted-2H-indazoles via a copper-catalyzed regioselective cross-coupling reaction. Org. Biomol. Chem. 2018, 16, 1816-1822. [CrossRef]

52. Nykaza, T.V.; Harrison, T.S.; Ghosh, A.; Putnik, R.A.; Radosevich, A.T. A biphilic phosphetane catalyzes N-N bond-forming Cadogan heterocyclization via PIII/PV=O redox cycling. J. Am. Chem. Soc. 2017, 139, 6839-6842. [CrossRef] [PubMed]

53. González-Medina, M.; Méndez-Lucio, O.; Medina-Franco, J.L. Activity landscape plotter: A web-based application for the analysis of structure-activity relationships. J. Chem. Inf. Model. 2017, 57, 397-402. [CrossRef] [PubMed]

54. Durant, J.L.; Leland, B.A.; Henry, D.R.; Nourse, J.G. Reoptimization of MDL keys for use in drug discovery. J. Chem. Inf. Comput. Sci. 2002, 42, 1273-1280. [CrossRef] [PubMed]

55. Rogers, D.; Hahn, M. Extended-connectivity fingerprints. J. Chem. Inf. Model. 2010, 50, 742-754. [CrossRef] [PubMed] 